\title{
HIGHER INTEGRABILITY FOR PARABOLIC SYSTEMS WITH NON-STANDARD GROWTH AND DEGENERATE DIFFUSIONS
}

\author{
Verena Bögelein ANd Frank DuZaAR
}

Abstract

The aim of this paper is to establish a Meyer's type higher integrability result for weak solutions of possibly degenerate parabolic systems of the type

$$
\partial_{t} u-\operatorname{div} a(x, t, D u)=\operatorname{div}\left(|F|^{p(x, t)-2} F\right) .
$$

The vector-field $a$ is assumed to fulfill a non-standard $p(x, t)$ growth condition. In particular it is shown that there exists $\varepsilon>0$ depending only on the structural data such that there holds:

$$
|D u|^{p(\cdot)(1+\varepsilon)} \in L_{\mathrm{loc}}^{1},
$$

together with a local estimate for the $p(\cdot)(1+\varepsilon)$-energy.

\section{Introduction}

In this paper we establish higher integrability properties of solutions to second order parabolic systems with non-standard $p(z)$-growth and possibly degenerate diffusion of the type:

$$
\partial_{t} u-\operatorname{div} a(z, D u)=\operatorname{div}\left(|F|^{p(z)-2} F\right) \quad \text { in } \Omega_{T} .
$$

Here, $\Omega_{T}$ denotes the space time cylinder $\Omega \times(0, T)$ over a bounded domain $\Omega \subseteq \mathbb{R}^{n}$ with dimension $n \geq 2$. We write $z=(x, t)$ for points in $\mathbb{R}^{n+1}, D u$ for the spatial gradient of $u$ and $\partial_{t} u$ for the derivative with respect to time. The function $u: \Omega_{T} \rightarrow \mathbb{R}^{N}$ with $N \geq 1$ can possibly be vector valued, so that we include in our considerations the case of parabolic systems. The precise structural assumptions on the vector-field $a$ and the exponent function $p(\cdot)$ will be presented later in Section 2, but the principal prototype we have in mind is the parabolic

2000 Mathematics Subject Classification. 35D10, 35K40, 35K65.

Key words. Higher integrability, degenerate parabolic systems, non-standard growth condition, parabolic $p$-Laplacean. 
$p(z)$-Laplacean system:

$$
\partial_{t} u-\operatorname{div}\left(|D u|^{p(z)-2} D u\right)=\operatorname{div}\left(|F|^{p(z)-2} F\right) \quad \text { in } \Omega_{T} .
$$

As usual we consider weak solutions $u$ of (1.1), meaning that they belong to a certain parabolic Sobolev space inheriting the growth of the vectorfield $a$; the precise definition of a weak (energy) solution will be given in Section 2.

As already mentioned above the primary purpose of this paper is to establish a Meyer's type higher integrability result for the spatial gradient of weak solutions of parabolic systems as in (1.1); to be more precise it will be shown that there exists a constant $\varepsilon>0$, depending only on the structural data and on the degree of integrability of the right-hand side $F$ such that

$$
|D u|^{p(\cdot)(1+\varepsilon)} \in L_{\mathrm{loc}}^{1}\left(\Omega_{T}\right) .
$$

In addition to the previous qualitative higher integrability a precise quantitative estimate is established. Therefore our result ensures that weak solutions of (1.1) belong to a slightly higher Sobolev space than the natural energy space determined uniquely by the growth of the vector-field $a$ and therefore obey a certain self-improving property of integrability. This is the $p(z)$-analogue of the higher integrability result of Kinunnen and Lewis $[\mathbf{2 9}]$ concerning parabolic systems with a standard $p$-growth condition. Moreover, it generalizes the result of Antontsev and Zhikov $[\mathbf{6}]$ for homogeneous $p(z)$-Laplacean equations to the case of general systems considered in (1.1).

1.1. A short introduction to the self-improving property of integrability. For elliptic equations and systems with constant polynomial growth $p \equiv$ const $\in(1, \infty)$ it is a by now classical fact that weak solutions are locally (and, depending on the boundary data, globally) higher integrable in the sense mentioned before and therefore admit the self-improving property of integrability. This was first observed for the Jacobian of a quasi conformal mapping by Gehring [21] and later on for solutions to elliptic systems by Meyers and Elcrat [35] (see also [23] and the monograph $[\mathbf{2 2}]$ for further references therein). In principle the argument of the proof is based on certain reverse Hölder inequalities and an application of Gehring's lemma. Such reverse Hölder inequalities are typically derived from a Caccioppoli type inequality - an estimate having the structure of a reverse Poincaré inequality - followed by an application of Sobolev-Poincaré's inequality to the right-hand side. The first treatment of the higher integrability for solutions to elliptic 
equations with non-standard $p(x)$-growth goes back to Zhikov [41]. Improvements of this result can be found for instance in [13], [42]. At this stage we point out that a complete Calderón-Zygmund theory for the $p$ Laplacean equation was essentially obtained by Iwaniec [27], while the vectorial case, i.e. the case of systems, was treated by DiBenedetto and Manfredi [18]. For similar results concerning equations with VMO-coefficients we refer to $[\mathbf{3 1}],[\mathbf{3 2}]$ and the references therein. Finally, a complete Calderón-Zygmund theory for equations with non-standard $p(x)$-growth was achieved by Acerbi and Mingione [3]. In their proof the higher integrability of the solutions plays an important role.

The main idea to deal with the non-standard $p(x)$-growth condition, which leads to non-homogeneous estimates, is a localization technique that allows to homogenize the estimates. In this way one obtains a reverse Hölder type inequality which is comparable to the one from the constant growth case and which is the key to the higher integrability.

In the parabolic setting the elliptic techniques to prove higher integrability cannot be applied directly for several obstructions. Already in the simplest case of linear growth $p \equiv 2$ the application of a SobolevPoincaré inequality is not possible due to the definition of a weak solution which a priori admits no regularity with respect to time. The second main obstruction in the case $p \equiv$ const $\neq 2$ results from the nonhomogeneous scaling of the parabolic system in the sense that a solution of the parabolic $p$-Laplacean system does not remain a solution under multiplication by a constant different form 0 and 1 . However, reverse Hölder inequalities should take a form which persists invariant under scaling. Therefore, one is forced to choose a system of parabolic cylinders different from the standard ones whose space-time scaling depends on the local behavior of the solution itself, and which, in a certain sense, re-balances the non-homogeneous scaling of the parabolic system. Such kind of cylinders were introduced in the pioneering work of DiBenedetto and Friedman $[\mathbf{1 4}],[\mathbf{1 5}],[\mathbf{1 6}],[\mathbf{1 7}]$. The strategy is to find parabolic cylinders of the form

(1.3) $Q_{\varrho}^{(\lambda)}\left(z_{0}\right):=B_{\varrho}\left(x_{0}\right) \times\left(t_{0}-\lambda^{2-p} \varrho^{2}, t_{0}+\lambda^{2-p} \varrho^{2}\right), \quad z_{0} \equiv\left(x_{0}, t_{0}\right)$,

such that the scaling parameter $\lambda>0$ and the average of $|D u|^{p}$ over $Q_{\varrho}^{(\lambda)}\left(z_{0}\right)$ are coupled in the following way:

$$
f_{Q_{\varrho}^{(\lambda)}\left(z_{0}\right)}|D u|^{p} d z \approx \lambda^{p} .
$$

Such cylinders are called intrinsic cylinders or cylinders with intrinsic coupling. The delicate aspect in this coupling clearly relies in the 
fact that the value of the integral average must be comparable to the scaling factor, which itself is involved in the construction of its support. On such intrinsic cylinders, i.e. when $|D u|$ is comparable to $\lambda$ in the above sense, the parabolic $p$-Laplacean system $\partial_{t} u=\operatorname{div}\left(|D u|^{p-2} D u\right)$ behaves heuristically like $\partial_{t} u=\lambda^{p-2} \Delta u$. Therefore, using intrinsic cylinders of the type $Q_{\varrho}^{(\lambda)}\left(z_{0}\right)$ we can re-balance the occurring multiplicative factor $\lambda^{p-2}$, which has the same effect as re-scaling $u$ in time by a factor $\lambda^{2-p}$. Finally, the construction of such intrinsic cylinders is a delicate issue and can be achieved in the context of higher integrability by certain stopping time arguments. These techniques allow the proof of a reverse Hölder type inequality on such intrinsic cylinders, which together with suitable covering arguments yield the higher integrability.

The case of linear growth $p \equiv 2$ was treated by Giaquinta and Struwe [24]. Note that this case is much easier since it falls into the realm of non-degenerate problems which can be treated without using the intrinsic geometry. The case $\frac{2 n}{n+2}<p \neq 2$ goes back to the work of Kinnunen and Lewis $[\mathbf{2 9}]$, and for a global version we refer to $[\mathbf{3 8}]$. The restriction on the growth exponent from below is unavoidable and already occurs in the parabolic regularity theory for $p$-Laplacean systems and equations; see [14]. Finally, the case of higher order parabolic systems with degenerate diffusions was treated in $[\mathbf{7}],[\mathbf{1 1}]$.

For differentiable vector-fields, i.e. vector-fields being differentiable with respect to $x$ - or at least Lipschitz continuous with respect to $x$ higher integrability results can be found in [5], [19], [39]. Under such an assumption it is possible to differentiate the system with respect to $x$, establishing the existence of second order spatial derivatives, which when combined with a certain parabolic embedding yields a reverse Hölder inequality. Note that this technique produces an explicit higher integrability exponent. For the case $p=2$ a global version valid up to the lateral and initial boundary was achieved in $[\mathbf{9}]$; note that the proof of the lateral boundary version is much more delicate, since one can differentiate the system only with respect to the tangential spatial derivatives.

We should also mention that a complete Calderón-Zygmund theory for degenerate parabolic equations and for $p$-Laplacean type systems with a quasi-diagonal Uhlenbeck structure, i.e. systems of the type $\partial_{t} u-$ $\operatorname{div}(g(|D u|) D u)=\operatorname{div}\left(|F|^{p-2} F\right)$ where $g(|D u|) \approx|D u|^{p-2}$, has been established by Acerbi and Mingione in [4]; a corresponding result for general systems without a Uhlenbeck structure can be inferred from $[\mathbf{1 9}]$.

Finally, we should also mention related higher integrality results for very weak solutions to elliptic and parabolic systems. Here, the problem 
is to show that a very weak solution, i.e. a solution belonging to a slightly worse Sobolev space than the natural energy space (determined by the growth of the vector-field), actually belongs to the natural energy space (see [35], [28], [33] for the case of elliptic systems, [30], [8] for parabolic systems, and [12] for very weak solutions to elliptic systems with nonstandard $p(x)$-growth). The major difficulty in dealing with very weak solutions is that the solution multiplied by a cut-off function cannot be used as a testing function in the weak formulation of the system. This can be overcome by a variety of different techniques such as the nonlinear Hodge decomposition from [28] or a Whitney type construction of suitable test functions from $[\mathbf{3 3}],[\mathbf{3 0}]$. We note that the proof in the parabolic setting is again more delicate due to the missing regularity of solutions with respect to time and the non-homogeneous scaling behavior of the system as explained above.

The only higher integrability result available for parabolic problems with $p(z)$-growth is to our knowledge the result by Antontsev and Zhikov [6] which we already mentioned before. This result, however, is concerned with the pure homogeneous parabolic $p(z)$-Laplacean equation, i.e. (1.2) for the scalar case $N=1$ and $F \equiv 0$. We note that the basic difference with respect to the general case (1.1) is that solutions can be shown to be bounded (by a variant of Moser's iteration scheme) and therefore certain integrability exponents occurring in the course of the proofs can be diminished. Moreover, in contrast to our result the constants in the final quantitative higher integrability estimate depend on the solution itself via the $L^{\infty}-L^{2}$ - norm of $u$ and the $L^{p(\cdot)}$-energy of $D u$. In particular, the improvement in the integrability exponent depends on the solution itself, while in the main result of our paper, i.e. in Theorem 2.2 , the gain in integrability can be determined in a universal way in dependence of the structural assumptions and is therefore independent of the solution itself. The only dependence on the solution occurs in the size of the cylinders on which the quantitative higher integrability estimate is established. The fact that the integrability exponent does not depend on the solution is even new for the corresponding elliptic case, to which our technique certainly also applies. It is also worth to mention that our methods allow the treatment of general parabolic systems with $p(z)$-growth, i.e. the vector valued case $N>1$, with non-vanishing right-hand sides.

Our result can be helpful in different directions, since the starting point of any localization procedure in the regularity theory for parabolic systems with non-standard $p(z)$-growth will be a suitable localization technique based on the higher integrability. Therefore, possible 
applications are a partial regularity theory for general parabolic systems with $p(z)$-growth without degeneration (cf. [1], [2] for the elliptic case), the $C^{1, \alpha}$-regularity for degenerate parabolic systems with quasi-diagonal structure, i.e. a structure prescribing that the gradient non-linearities depend only on the explicit scalar quantity $|D u|$, or a related partial regularity theory for parabolic systems with degenerate $p(z)$-diffusion (cf. $[\mathbf{1 4}],[\mathbf{1 0}]$ for related results in the case $p \equiv$ const).

1.2. Plan of the paper. The paper is structured as follows. In Section 2 we give the precise assumptions for the vector-field $a$, the definition of a weak solution to (1.1) and the statement of the main higher integrability result. Section 3 includes the technical preliminaries as well as a description of the intrinsic geometry and a localization principle. In the subsequent Section 4 we give the proof of the Cacciopoli-inequality for solutions of parabolic $p(z)$-systems. This is formulated on general parabolic cylinders of the type

$$
Q_{\varrho}^{(\lambda)}\left(z_{0}\right):=B_{\varrho}\left(x_{0}\right) \times\left(t_{0}-\lambda^{\frac{2-p\left(z_{0}\right)}{p\left(z_{0}\right)}} \varrho^{2}, t_{0}+\lambda^{\frac{2-p\left(z_{0}\right)}{p\left(z_{0}\right)}} \varrho^{2}\right)
$$

without assuming a priori an intrinsic coupling. At a first glance this looks very much the same as a formulation on parabolic cylinders from (1.3) as one could substitute $\lambda$ by $\lambda^{\frac{1}{p\left(z_{0}\right)}}$. Anyhow, in contrast to the cylinders defined in (1.3) and used for the case $p \equiv$ const, now the scaling factor $\lambda^{\frac{2-p\left(z_{0}\right)}{p\left(z_{0}\right)}}$ depends on the center $z_{0}$ of the cylinder and therefore varies from point to point. Moreover, we use this non-standard scaling factor in the definition of the intrinsic cylinders with intrinsic coupling as follows:

$$
f_{Q_{\varrho}^{(\lambda)}\left(z_{0}\right)}|D u|^{p(\cdot)} d z \approx \lambda .
$$

This has the advantage that the right-hand side is independent of $p\left(z_{0}\right)$ and this is crucial for the multiplicative gain in the integrability exponent. Section 5 is now devoted to the derivation of suitable Poincare and Sobolev-Poincaré type inequalities which are necessary to obtain the reverse Hölder type inequality. Again the Poincaré inequality is established on general parabolic cylinders, while for the Sobolev-Poincaré inequality one has to work under the assumption of an intrinsic coupling. The latter assumption is necessary in order to re-balance the inhomogeneous scaling behavior of the system. In Section 6 we give the proof of the reverse Hölder type inequality on intrinsic cylinders. Section 7 is then dedicated to the proof of the main result. Therein, by use of a suitable stopping time argument we construct a covering of the super-level set of 
the spatial gradient by intrinsic cylinders on which the reverse Hölder inequality can be applied. Due to the non-standard growth condition the covering has to capture a situation with intrinsic scaling parameters varying with respect to space and time; i.e. the cylinders $Q_{\varrho}^{(\lambda)}\left(z_{0}\right)$ are re-scaled in time direction by the scaling factor $\lambda^{\frac{2-p\left(z_{0}\right)}{p\left(z_{0}\right)}}$ depending additionally on the center $z_{0}$ of the intrinsic cylinder. This makes necessary the use of a Vitali-type covering for a collection of non-uniformly scaled intrinsic cylinders. However, the global energy bound yields an upper bound for the size of the radii, a crucial fact used in the proof of the Vitali-type covering lemma; see Lemma 7.1. In this respect the proof of the main theorem differs form the known ones of the previous higher integrability results. Moreover, instead of working globally with a re-scaled function of the type $d_{\mathcal{P}}\left(z, \partial Q_{\varrho}\right)^{\alpha}|D u|^{p(z)}$, with a suitable exponent $\alpha$, on the parabolic cylinder $Q_{\varrho}$ in question - which allows to re-absorb a certain integral - we work directly with $|D u|^{p(z)}$ on nested cylinders $Q_{\varrho / 2} \subseteq Q_{t} \subset Q_{s} \subseteq Q_{\varrho}$. This idea was first successfully applied in the context of measure data problems in [36]. This allows to re-absorb, roughly speaking, an integral of the form $\int_{Q_{s}}|D u|^{p(\cdot)(1+\varepsilon)} d z-$ which naturally occurs in right-hand sides of the estimates in the course of the proof - in the left-hand side. Thereby, the problem that such integrals are a priori not known to be finite, can be resolved as usual by a truncation argument. This is one of the technical novelties of the paper and simplifies also the proof of higher integrability in the elliptic case. In the final Section 8 we indicate how the methods can be used to treat a right-hand side of the type $b(z, D u)$ with controllable $p(z)$-growth. By this we understand that $|b(z, w)| \lesssim|w|^{p(z)-1}$.

Acknowledgements. This work is supported by the ERC grant 207573 "Vectorial Problems". We would like to thank P. Baroni for useful comments on a preliminary version of the paper leading to a more neat formulation of the statement of the main result.

\section{Statements}

In the following $\Omega$ will denote a bounded domain in $\mathbb{R}^{n}$ with $n \geq 2$ and $\Omega_{T}:=\Omega \times(0, T), T>0$ will be the space-time cylinder over $\Omega$. Points in $\mathbb{R}^{n+1}$ will be denoted by $z=(x, t)$. Differentiation with respect to the spatial variables will be denoted by $D u$, while the differentiation with respect to time by $\partial_{t} u$ or $u_{t}$. The spaces $L^{p}\left(\Omega, \mathbb{R}^{N}\right)$ and $W^{1, p}\left(\Omega, \mathbb{R}^{N}\right)$ stand for the usual Lebesgue, respectively Sobolev spaces. Concerning parabolic cylinders we use symmetric parabolic cylinders of 
the form $Q_{\varrho}\left(z_{0}\right):=B_{\varrho}\left(x_{0}\right) \times\left(t_{0}-\varrho^{2}, t_{0}+\varrho^{2}\right)$ instead of the standard cylinders $B_{\varrho}\left(x_{0}\right) \times\left(t_{0}-\varrho^{2}, t_{0}\right)$. Here, $B_{\varrho}\left(x_{0}\right)$ denotes the ball of radius $\varrho>0$ with center $x_{0} \in \mathbb{R}^{n}$.

We shall consider vector fields $a: \Omega_{T} \times \mathbb{R}^{N n} \rightarrow \mathbb{R}^{N n}$ which are assumed to be Carathéodory regular and to satisfy the following non-standard $p(z)$-growth and ellipticity assumptions:

$$
|a(z, w)| \leq L(|w|+1)^{p(z)-1}, \quad a(z, w) \cdot w \geq \nu|w|^{p(z)},
$$

whenever $z \in \Omega_{T}$ and $w \in \mathbb{R}^{N n}$. Here, $0<\nu \leq 1 \leq L$ are fixed structural parameters. The exponent function $p: \Omega_{T} \rightarrow\left(\frac{2 n}{n+2}, \infty\right)$ is assumed to be continuous with a modulus of continuity $\omega:[0, \infty) \rightarrow[0,1]$. More precisely, we shall assume that $\omega$ is a concave, non-decreasing function with $\lim _{s \downarrow 0} \omega(s)=0=\omega(0)$ and that there exist constants $\gamma_{1}, \gamma_{2}<\infty$ such that

$$
\frac{2 n}{n+2}<\gamma_{1} \leq p(z) \leq \gamma_{2} \quad \text { and } \quad\left|p\left(z_{1}\right)-p\left(z_{2}\right)\right| \leq \omega\left(d_{\mathcal{P}}\left(z_{1}, z_{2}\right)\right),
$$

holds for any choice of $z_{1}, z_{2} \in \Omega_{T}$. Here the parabolic distance is given by

$$
d_{\mathcal{P}}\left(z_{1}, z_{2}\right):=\max \left\{\left|x_{1}-x_{2}\right|, \sqrt{\left|t_{1}-t_{2}\right|}\right\},
$$

for $z_{1}=\left(x_{1}, t_{1}\right), z_{2}=\left(x_{2}, t_{2}\right) \in \mathbb{R}^{n+1}$. At this stage it is worth to mention that assuming the existence of such $\gamma_{1}, \gamma_{2}$ is not restrictive since the result we are going to prove is of local nature. We mention that the previous lower bound on $\gamma_{1}$ is a typical assumption in the regularity theory for non-linear parabolic equations and systems. Finally, for the modulus of continuity $\omega$ we assume the following weak logarithmic continuity condition to hold:

$$
\limsup _{\varrho \downarrow 0} \omega(\varrho) \log \left(\frac{1}{\varrho}\right)<+\infty .
$$

By virtue of (2.4) we may assume for a constant $L_{1}>0$ depending on $\omega(\cdot)$ that

$$
\omega(\varrho) \log \left(\frac{1}{\varrho}\right) \leq L_{1}, \quad \text { for all } \varrho \in(0,1] .
$$

For $k \in \mathbb{N}$ we define $L^{p(\cdot)}\left(\Omega_{T}, \mathbb{R}^{k}\right)$ to be the set of those measurable functions $v: \Omega_{T} \rightarrow \mathbb{R}^{k}$ such that $|v|^{p(\cdot)} \in L^{1}\left(\Omega_{T}\right)$; i.e.

$$
L^{p(\cdot)}\left(\Omega_{T}, \mathbb{R}^{k}\right):=\left\{v: \Omega_{T} \rightarrow \mathbb{R}^{k}: \int_{\Omega_{T}}|v|^{p(\cdot)} d z<+\infty\right\} .
$$

Finally, for the right-hand side we assume $F \in L^{p(\cdot)}\left(\Omega_{T}, \mathbb{R}^{N n}\right)$, at least. We are now ready to give the definition of a weak solution to the nonlinear parabolic system (1.1): 
Definition 2.1. We identify a function $u \in L^{1}\left(\Omega_{T}, \mathbb{R}^{N}\right)$ as a weak solution of the parabolic system (1.1) if and only if $u \in L^{2}\left(\Omega_{T}, \mathbb{R}^{N}\right)$, $D u \in L^{p(\cdot)}\left(\Omega_{T}, \mathbb{R}^{N n}\right)$ and

$$
\int_{\Omega_{T}}\left[u \cdot \varphi_{t}-a(z, D u) \cdot D \varphi\right] d z=\int_{\Omega_{T}}|F|^{p(\cdot)-2} F \cdot D \varphi d z
$$

holds, whenever $\varphi \in C_{0}^{\infty}\left(\Omega_{T}, \mathbb{R}^{N}\right)$.

The following theorem is our main result:

Theorem 2.2. Let $\sigma>0$ and $p: \Omega_{T} \rightarrow\left[\gamma_{1}, \gamma_{2}\right]$ satisfy (2.2) and (2.4). Then, there exists $\varepsilon_{0}=\varepsilon_{0}\left(n, N, \nu, L, L_{1}, \gamma_{1}, \gamma_{2}, \sigma\right) \in(0, \sigma]$ such that the following holds: Whenever $u$ is a weak solution of the parabolic system (1.1), where (2.1) is in force and $F \in L^{p(\cdot)(1+\sigma)}\left(\Omega_{T}, \mathbb{R}^{N n}\right)$ then

$$
|D u|^{p(\cdot)\left(1+\varepsilon_{0}\right)} \in L_{\mathrm{loc}}^{1}\left(\Omega_{T}\right) .
$$

Moreover, for $M \geq 1$ there exists a radius $r_{0} \equiv r_{0}\left(n, L_{1}, \gamma_{1}, \gamma_{2}, M\right)>0$ such that there holds: If

$$
\int_{\Omega_{T}}(|D u|+|F|+1)^{p(\cdot)} d z \leq M
$$

and $\varepsilon \in\left(0, \varepsilon_{0}\right]$, then for any parabolic cylinder $Q_{2 r} \equiv Q_{2 r}\left(\mathfrak{z}_{0}\right) \subseteq \Omega_{T}$ with $r \in\left(0, r_{0}\right]$ there holds

$$
\begin{aligned}
f_{Q_{r}}|D u|^{p(\cdot)(1+\varepsilon)} d z \leq c\left(f_{Q_{2 r}}(|D u|\right. & \left.+|F|+1)^{p(\cdot)} d z\right)^{1+\varepsilon d} \\
& +c f_{Q_{2 r}}(|F|+1)^{p(\cdot)(1+\varepsilon)} d z
\end{aligned}
$$

for a constant $c=c\left(n, N, \nu, L, L_{1}, \gamma_{1}, \gamma_{2}\right)$. Here we used the abbreviation:

$$
d \equiv d\left(p_{0}\right):=\left\{\begin{array}{ll}
\frac{2 p_{0}}{p_{0}(n+2)-2 n}, & \text { if } p_{0}<2, \\
\frac{p_{0}}{2}, & \text { if } p_{0} \geq 2,
\end{array} \text { where } p_{0}:=p\left(\mathfrak{z}_{0}\right) .\right.
$$

\section{Preliminaries and notations}

3.1. Notations. As mentioned already in Section 2 we shall always write $z_{0}=\left(x_{0}, t_{0}\right)$ for a point $z_{0} \in \mathbb{R}^{n+1}$ and we shall consider - as we did for instance in the statement of Theorem 2.2- symmetric parabolic cylinders around $z_{0}$ of the form $Q_{\varrho}\left(z_{0}\right):=B_{\varrho}\left(x_{0}\right) \times\left(t_{0}-\varrho^{2}, t_{0}+\varrho^{2}\right)$. 
Moreover, in the course of the proof of our main result, in order to rebalance the non-homogeneity of the parabolic system, we shall deal with certain scaled cylinders of the form

$$
Q_{\varrho}^{(\lambda)}\left(z_{0}\right):=B_{\varrho}\left(x_{0}\right) \times \Lambda_{\varrho}^{(\lambda)}\left(z_{0}\right),
$$

where

$$
\Lambda_{\varrho}^{(\lambda)}\left(z_{0}\right):=\left(t_{0}-\lambda^{\frac{2-p_{0}}{p_{0}}} \varrho^{2}, t_{0}+\lambda^{\frac{2-p_{0}}{p_{0}}} \varrho^{2}\right) .
$$

In any case, when considering a certain cylinder $Q_{\varrho}^{(\lambda)}\left(z_{0}\right)$ with center $z_{0}$ by $p_{0}$ we denote the value of $p(\cdot)$ at the center of the cylinder, i.e. $p_{0} \equiv$ $p\left(z_{0}\right)$. Note that such a system of scaled cylinders is non-uniform in the sense that the scaling $\lambda^{\frac{2-p_{0}}{p_{0}}}$ depends on the particular point $z_{0}$ via $p_{0} \equiv$ $p\left(z_{0}\right)$. Moreover, we denote by $p_{1}, p_{2}$ the infimum resp. supremum of $p(\cdot)$ over the intrinsic cylinder $Q_{\varrho}^{(\lambda)}\left(z_{0}\right)$, i.e.

$$
p_{1} \equiv \inf _{Q_{\varrho}^{(\lambda)}\left(z_{0}\right)} p(\cdot), \quad p_{2} \equiv \sup _{Q_{\varrho}^{(\lambda)}\left(z_{0}\right)} p(\cdot) .
$$

In any case, where it is clear from the context which cylinder is meant, we refer to this notation; otherwise we shall give precise definitions.

3.2. Preliminaries. In order to "re-absorb" certain terms, we will use the following iteration lemma, which is a standard tool and can for instance be found in $[\mathbf{2 2}]$.

Lemma 3.1. Let $0<\vartheta<1, A, C \geq 0$ and $\beta>0$. Then there exists a constant $c=c(\beta, \vartheta)$ such that there holds: For any non-negative bounded function satisfying

$$
\phi(t) \leq \vartheta \phi(s)+A(s-t)^{-\beta}+C \quad \text { for all } 0<r \leq t<s \leq \varrho,
$$

we have

$$
\phi(r) \leq c\left[A(\varrho-r)^{-\beta}+C\right] .
$$

Finally, we state Gagliardo-Nirenberg's inequality in a form which shall be suitable for our purposes later.

Lemma 3.2. Let $B_{\varrho}\left(x_{0}\right) \subset \mathbb{R}^{n}$ with $0<\varrho \leq 1,1 \leq \sigma, q, r<\infty$ and $\vartheta \in(0,1)$ such that $-\frac{n}{\sigma} \leq \vartheta\left(1-\frac{n}{q}\right)-(1-\vartheta) \frac{n}{r}$. Then there exists a constant $c=c(n, \sigma)$ such that for any $u \in W^{1, q}\left(B_{\varrho}\left(x_{0}\right)\right)$ there holds:

$$
f_{B_{\varrho}\left(x_{0}\right)}\left|\frac{u}{\varrho}\right|^{\sigma} d x \leq c\left(f_{B_{\varrho}\left(x_{0}\right)}\left|\frac{u}{\varrho}\right|^{q}+|D u|^{q} d x\right)^{\frac{\vartheta \sigma}{q}}\left(f_{B_{\varrho}\left(x_{0}\right)}\left|\frac{u}{\varrho}\right|^{r} d x\right)^{\frac{(1-\vartheta) \sigma}{r}} .
$$


3.3. Intrinsic geometry and localization. In order to re-balance the non-homogeneous behavior of the parabolic system we shall use the so called intrinsic geometry which - in the case of standard growth conditions - was introduced by DiBenedetto [14], [15], [16], [17]. More precisely, denoting by $u$ a weak solution of (1.1) in $\Omega_{T}$, a cylinder

$$
Q_{\varrho}^{(\lambda)}\left(z_{0}\right) \equiv B_{\varrho}\left(x_{0}\right) \times\left(t_{0}-\lambda^{\frac{2-p_{0}}{p_{0}}} \varrho^{2}, t_{0}+\lambda^{\frac{2-p_{0}}{p_{0}}} \varrho^{2}\right) \subseteq \Omega_{T}
$$

with $p_{0} \equiv p\left(z_{0}\right)$ is called intrinsic if the following property holds (in a quantifiable sense):

$$
f_{Q_{\varrho}^{(\lambda)}\left(z_{0}\right)}(|D u|+|F|+1)^{p(z)} d z \approx \lambda .
$$

On such cylinders we can prove homogeneous estimates although the parabolic system (1.1) is non-homogeneous. In order to deal with the non-standard growth property of the vector field $a(\cdot)$ we shall use - as it is usual in the elliptic case - suitable localization arguments, i.e. arguments employing the fact that locally the system behaves like a system of constant $p$-growth modulo small perturbations. These localization arguments have to be combined with the intrinsic geometry and this makes the problem technically much more involved than the corresponding elliptic one. In the following we shall briefly explain the argument. Suppose that on some cylinder $Q_{\varrho}^{(\lambda)}\left(z_{0}\right) \subseteq \Omega_{T}$ with $0<\varrho \leq 1$ and $\lambda \geq 1$ we have the following intrinsic coupling:

$$
\lambda \leq \kappa \int_{Q_{\varrho}^{(\lambda)}\left(z_{0}\right)}(|D u|+|F|+1)^{p(z)} d z \quad \text { for some } \kappa \geq 1 .
$$

Then, using $\left|Q_{\varrho}^{(\lambda)}\left(z_{0}\right)\right|=2 \alpha_{n} \varrho^{n+2} \lambda^{\frac{2-p_{0}}{p_{0}}}$ (where $\alpha_{n}$ denotes the measure of the unit ball in $\mathbb{R}^{n}$ ) and (2.7) in (3.1) we find

$$
\lambda^{\frac{2}{p_{0}}} \leq \frac{\kappa}{2 \alpha_{n} \varrho^{n+2}} \int_{Q_{\varrho}^{(\lambda)}\left(z_{0}\right)}(|D u|+|F|+1)^{p(z)} d z \leq \frac{c(n) \kappa M}{\varrho^{n+2}} .
$$

This immediately leads us to a bound of $\lambda$ in terms of $\varrho$ and the energy bound $M$; more precisely with a constant $\beta_{n} \equiv \beta_{n}(n) \geq 1$ we get:

$$
\lambda \leq\left(\frac{\beta_{n} \kappa M}{\varrho^{n+2}}\right)^{\frac{p_{0}}{2}}
$$

On $Q_{\varrho}^{(\lambda)}\left(z_{0}\right)$ we now define

$$
p_{1}:=\inf _{Q_{\varrho}^{(\lambda)}\left(z_{0}\right)} p(\cdot) \quad \text { and } \quad p_{2}:=\sup _{Q_{\varrho}^{(\lambda)}\left(z_{0}\right)} p(\cdot) .
$$


Then, from (2.2), the fact that $\lambda \geq 1$ and $p_{0} \geq \gamma_{1}$ we obtain the following preliminary bound for the oscillation of $p(\cdot)$ on $Q_{\varrho}^{(\lambda)}\left(z_{0}\right)$ :

$$
p_{2}-p_{1} \leq \omega\left(2 \varrho+\sqrt{2 \lambda^{\frac{2-p_{0}}{p_{0}}} \varrho^{2}}\right)
$$

In the case $p_{0} \geq 2$ this leads us to

$$
p_{2}-p_{1} \leq \omega(4 \varrho),
$$

while in the case $\frac{2 n}{n+2}<p_{0}<2$ we infer from (3.2) that

$$
\begin{aligned}
p_{2}-p_{1} & \leq \omega\left(4 \lambda^{\frac{2-p_{0}}{2 p_{0}}} \varrho\right) \leq \omega\left(4\left(\beta_{n} \kappa M\right)^{\frac{2-\gamma_{1}}{4}} \varrho^{1-\frac{\left(2-\gamma_{1}\right)(n+2)}{4}}\right) \\
& \leq \omega\left(4 \sqrt{\beta_{n} \kappa M} \varrho^{\gamma_{1} \frac{n+2}{4}-\frac{n}{2}}\right)=\omega\left(\Gamma \varrho^{\gamma_{1} \frac{n+2}{4}-\frac{n}{2}}\right)
\end{aligned}
$$

where we have defined $\Gamma:=4 \sqrt{\beta_{n} \kappa M} \geq 4$. Note that the restriction $\gamma_{1}>$ $\frac{2 n}{n+2}$ ensures that the exponent of $\varrho$ is positive, i.e. $\gamma_{1} \frac{n+2}{4}-\frac{n}{2}>0$. Combining the estimates from the cases $\gamma_{1} \geq 2$ and $\gamma_{1}<2$ we finally arrive at:

$$
p_{2}-p_{1} \leq \omega\left(\Gamma \varrho^{\alpha}\right), \quad \text { where } \quad \alpha:=\min \left\{1, \gamma_{1} \frac{n+2}{4}-\frac{n}{2}\right\} .
$$

Using the preceding inequality and the logarithmic bound (2.5) we infer that

$$
\begin{aligned}
\varrho^{-\left(p_{2}-p_{1}\right)} \leq \varrho^{-\omega\left(\Gamma \varrho^{\alpha}\right)} & =\exp \left[\omega\left(\Gamma \varrho^{\alpha}\right) \log \frac{1}{\varrho}\right]=\exp \left[\alpha^{-1} \omega\left(\Gamma \varrho^{\alpha}\right) \log \frac{1}{\varrho^{\alpha}}\right] \\
& =\exp \left[\alpha^{-1} \omega\left(\Gamma \varrho^{\alpha}\right)\left(\log \frac{1}{\Gamma \varrho^{\alpha}}+\log \Gamma\right)\right] \\
& \leq \mathrm{e}^{\frac{L_{1}}{\alpha}} \exp \left[\alpha^{-1} \omega\left(\Gamma \varrho^{\alpha}\right) \log \Gamma\right] .
\end{aligned}
$$

At this point we choose

$$
\varrho_{0} \leq \Gamma^{-\frac{2}{\alpha}} \equiv\left(4 \sqrt{\beta_{n} \kappa M}\right)^{-\frac{2}{\alpha}} .
$$

Keeping in mind the definition of $\alpha$ this determines $\varrho_{0}$ as a constant depending on $n, \gamma_{1}, \kappa, M$. Now, we assume that $0<\varrho \leq \varrho_{0}$ and use (2.5) again to estimate further

$$
\begin{aligned}
\varrho^{-\left(p_{2}-p_{1}\right)} & \leq \mathrm{e}^{\frac{L_{1}}{\alpha}} \exp \left[\alpha^{-1} \omega\left(\Gamma \varrho_{0}^{\alpha}\right) \log \Gamma\right] \\
& \leq \mathrm{e}^{\frac{L_{1}}{\alpha}} \exp \left[\alpha^{-1} \omega\left(\frac{1}{\Gamma}\right) \log \Gamma\right] \leq \mathrm{e}^{\frac{2 L_{1}}{\alpha}}
\end{aligned}
$$


Together with (3.2), the definition of $\Gamma,(3.3),(3.4)$ and (2.5) this finally implies

$$
\begin{aligned}
\lambda^{\frac{p_{2}-p_{1}}{p_{0}}} & \leq\left(\Gamma \varrho^{-\frac{n+2}{2}}\right)^{p_{2}-p_{1}} \leq \Gamma^{\omega\left(\Gamma \varrho_{0}^{\alpha}\right)} \mathrm{e}^{\frac{L_{1}(n+2)}{\alpha}} \\
& \leq \Gamma^{\omega\left(\Gamma^{-1}\right)} \mathrm{e}^{\frac{L_{1}(n+2)}{\alpha}} \leq \mathrm{e}^{\frac{L_{1}(n+2)}{\alpha}+L_{1}} \leq \mathrm{e}^{\frac{4 n L_{1}}{\alpha}} .
\end{aligned}
$$

3.4. Steklov averages. Since weak solutions of parabolic systems do not necessarily admit a time derivative in $L^{2}$ it is in general not possible to test the systems with the solution itself. To overcome this problem it is a standard tool in the theory of parabolic systems to use a certain mollification with respect to time when testing the parabolic system. Particularly the so called Steklov averages serve as such a suitable mollification. The precise definition is as follows: Given a function $f \in$ $L^{1}\left(\Omega_{T}\right)$ and $0<h<T$ the Steklov-mean $[f]_{h}$ of $f$ is defined by

$$
[f]_{h}(x, t) \equiv \begin{cases}\frac{1}{h} \int_{t}^{t+h} f(x, \tau) d \tau, & t \in(0, T-h), \\ 0, & t \in(T-h, T) .\end{cases}
$$

By use of Steklov-means of the solution $u$ the parabolic system (1.1) can be re-written in the following equivalent formulation (cf. [14, Chapter II]):

(3.7) $\int_{\Omega}\left(\partial_{t}[u]_{h} \cdot \varphi+[a(\cdot, D u)]_{h} \cdot D \varphi\right)(\cdot, t) d x=-\int_{\Omega}\left[|F|^{p(\cdot)-2} F\right]_{h}(\cdot, t) \cdot D \varphi d x$ whenever $\varphi \in W_{0}^{1, p(\cdot)}\left(\Omega, \mathbb{R}^{N}\right) \cap L^{2}\left(\Omega, \mathbb{R}^{N}\right)$.

\section{Caccioppoli inequality}

It is a well know fact that the first step towards a higher integrability result for solutions to elliptic and parabolic systems is a suitable Caccioppoli-type inequality (see for example $[\mathbf{2 9}]$, [30], [7], [8]). Such inequalities when combined with Sobolev-type estimates yield a reverse Hölder-type inequality on intrinsic parabolic cylinders.

Lemma 4.1. Suppose that $u$ is a weak solution of (1.1) in $\Omega_{T}$ under the assumptions $(2.1)$ and let $F \in L^{p(\cdot)}\left(\Omega_{T}, \mathbb{R}^{N n}\right)$. Then, there exists a constant $c=c\left(\nu, L, \gamma_{1}, \gamma_{2}\right)$ such that for all parabolic cylinders $Q_{\varrho}^{(\lambda)}\left(z_{0}\right) \subseteq \Omega_{T}$ with $0<\varrho \leq 1, \lambda>0$ and for all $r \in[\varrho / 2, \varrho)$ and 
$A \in \mathbb{R}^{N}$ there holds:

$$
\begin{aligned}
& \sup _{t \in \Lambda_{r}^{(\lambda)}\left(z_{0}\right)} f_{B_{r}\left(x_{0}\right)} \lambda^{\frac{p_{0}-2}{p_{0}}}\left|\frac{u(\cdot, t)-A}{r}\right|^{2} d x+f_{Q_{r}^{(\lambda)}\left(z_{0}\right)}|D u|^{p(\cdot)} d z \\
& \leq c f_{Q_{\varrho}^{(\lambda)}\left(z_{0}\right)}\left[\lambda^{\frac{p_{0}-2}{p_{0}}}\left|\frac{u-A}{\varrho-r}\right|^{2}+\left|\frac{u-A}{\varrho-r}\right|^{p(\cdot)}+|F|^{p(\cdot)}+1\right] d z .
\end{aligned}
$$

Proof: In our notation we shall omit the reference point $z_{0}$ writing for instance $Q_{\varrho}^{(\lambda)}$ instead of $Q_{\varrho}^{(\lambda)}\left(z_{0}\right)$. Next, we choose two cut-off functions $\eta \in C_{0}^{\infty}\left(B_{\varrho}\right)$ and $\zeta \in C^{1}(\mathbb{R})$ satisfying $0 \leq \eta \leq 1, \eta \equiv 1$ in $B_{r}$, $|\nabla \eta| \leq c /(\varrho-r)$ and $0 \leq \zeta \leq 1, \zeta \equiv 0$ on $\left(-\infty,-\lambda^{\frac{2-p_{0}}{p_{0}}} \varrho^{2}\right], \zeta \equiv 1$ on $\left[-\lambda^{\frac{2-p_{0}}{p_{0}}} r^{2}, \infty\right), 0 \leq \zeta^{\prime} \leq 2 \lambda^{\frac{p_{0}-2}{p_{0}}} /\left(\varrho^{2}-r^{2}\right)$. Choosing in the Steklovformulation (3.7) (for a.e. $\tau \in \Lambda_{\varrho}^{(\lambda)}$ ) the test-function

$$
\varphi_{h} \equiv \eta^{\gamma_{2}} \zeta^{2}\left([u]_{h}-A\right)
$$

and taking into account that $\partial_{\tau} A=0$ we obtain

$$
\begin{aligned}
\int_{B_{\varrho}}\left(\partial_{\tau}\left([u]_{h}-A\right) \cdot \varphi_{h}+[a(\cdot, D u)]_{h} \cdot D \varphi_{h}\right)(\cdot, \tau) d x \\
=-\int_{B_{\varrho}}\left[|F|^{p(\cdot)-2} F\right]_{h}(\cdot, \tau) \cdot D \varphi_{h} d x
\end{aligned}
$$

for a.e. $\tau \in \Lambda_{\varrho}^{(\lambda)}$. Next, we integrate the preceding identity with respect to $\tau$ over $\Lambda_{\varrho, t}^{(\lambda)}:=\Lambda_{\varrho}^{(\lambda)} \cap\{\tau \in \mathbb{R}: \tau \leq t\}$, where $t \in \Lambda_{\varrho}^{(\lambda)}$. Since $\zeta\left(-\lambda^{\frac{2-p_{0}}{p_{0}}} \varrho^{2}\right)=0$, we obtain for the first term on the left-hand side for a.e. $t \in \Lambda_{\varrho}^{(\lambda)}$ that

$$
\begin{aligned}
\int_{\Lambda_{\varrho, t}^{(\lambda)}} & \int_{B_{\varrho}}\left(\partial_{\tau}[u]_{h}-A\right) \cdot \varphi_{h} d x d \tau \\
\quad= & \int_{Q_{\varrho, t}^{(\lambda)}} \frac{1}{2} \partial_{\tau}\left(\left|[u]_{h}-A\right|^{2} \zeta^{2}\right) \eta^{\gamma_{2}}-\left|[u]_{h}-A\right|^{2} \eta^{\gamma_{2}} \zeta \zeta^{\prime} d z \\
\quad= & \frac{1}{2} \int_{B_{\varrho}}\left|[u]_{h}(\cdot, t)-A\right|^{2} \eta^{\gamma_{2}} \zeta(t)^{2} d x-\int_{Q_{\varrho, t}^{(\lambda)}}\left|[u]_{h}-A\right|^{2} \eta^{\gamma_{2}} \zeta \zeta^{\prime} d z,
\end{aligned}
$$

with the obvious abbreviation $Q_{\varrho, t}^{(\lambda)}:=Q_{\varrho}^{(\lambda)} \cap\left\{(x, \tau) \in \mathbb{R}^{n+1}: \tau \leq t\right\}$. Inserting this above and passing to the limit $h \downarrow 0$ we find that for a.e. 
$t \in \Lambda_{\varrho}^{(\lambda)}$ there holds:

$$
\begin{aligned}
\frac{1}{2} \int_{B_{\varrho}}|u(\cdot, t)-A|^{2} \eta^{\gamma_{2}} \zeta(t)^{2} d x+\int_{Q_{e, t}^{(\lambda)}} a(\cdot, D u) \cdot D u \eta^{\gamma_{2}} \zeta^{2} d z \\
=\int_{Q_{e, t}^{(\lambda)}}\left[|u-A|^{2} \eta^{\gamma_{2}} \zeta \zeta^{\prime}-\gamma_{2} a(\cdot, D u) \cdot \nabla \eta \otimes(u-A) \eta^{\gamma_{2}-1} \zeta^{2}\right] d z \\
\quad-\int_{Q_{e, t}^{(\lambda)}}|F|^{p(\cdot)-2} F \cdot D \varphi d z .
\end{aligned}
$$

Here we defined $\varphi \equiv \eta^{\gamma_{2}} \zeta^{2}(u-A)$. Using the ellipticity and growth conditions for the vector field $a(\cdot)$ from (2.1), Young's inequality, $\zeta^{\prime} \leq$ $2 \lambda^{\frac{p_{0}-2}{p_{0}}} /\left(\varrho^{2}-r^{2}\right) \leq 2 \lambda^{\frac{p_{0}-2}{p_{0}}} /(\varrho-r)^{2},|\nabla \eta| \leq c /(\varrho-r)$ together with $0 \leq \eta, \zeta \leq 1$ and $\left(\gamma_{2}-1\right) \cdot \frac{p(z)}{p(z)-1} \geq \gamma_{2}$ we infer that

$$
\begin{aligned}
\frac{1}{2} \int_{B_{\varrho}} \mid & u(\cdot, t)-\left.A\right|^{2} \eta^{\gamma_{2}} \zeta^{2}(t) d x+\nu \int_{Q_{\varrho, t}^{(\lambda)}}|D u|^{p(\cdot)} \eta^{\gamma_{2}} \zeta^{2} d z \\
\leq & \int_{Q_{\varrho, t}^{(\lambda)}}\left[2 \lambda^{\frac{p_{0}-2}{p_{0}}}\left|\frac{u-A}{\varrho-r}\right|^{2}+c L \gamma_{2}(|D u|+1)^{p(\cdot)-1}\left|\frac{u-A}{\varrho-r}\right| \eta^{\gamma_{2}-1} \zeta^{2}\right] d z \\
& +\int_{Q_{\varrho, t}^{(\lambda)}}|F|^{p(\cdot)-1}\left[|D u| \eta^{\gamma_{2}} \zeta^{2}+c \gamma_{2}\left|\frac{u-A}{\varrho-r}\right|\right] d z \\
\leq & \frac{\nu}{2} \int_{Q_{\varrho, t}^{(\lambda)}}|D u|^{p(\cdot)} \eta^{\gamma_{2}} \zeta^{2} d z \\
& +c \int_{Q_{\varrho}^{(\lambda)}}\left[\lambda^{\frac{p_{0}-2}{p_{0}}}\left|\frac{u-A}{\varrho-r}\right|^{2}+\left|\frac{u-A}{\varrho-r}\right|^{p(\cdot)}+|F|^{p(\cdot)}+1\right] d z
\end{aligned}
$$

for a constant $c=c\left(\nu, L, \gamma_{1}, \gamma_{2}\right)$. At this stage we re-absorb as usual the first term of the right-hand side into the left-hand side. Recalling the choices of $\eta$ and $\zeta$, in particular that $\eta \equiv 1$ on $B_{r}$ and $\zeta \equiv 1$ on $\Lambda_{r}^{(\lambda)}$, we arrive - after taking mean values - at the following estimate valid for a.e. $t \in \Lambda_{r}^{(\lambda)}$ :

$$
\begin{aligned}
f_{B_{r}} \lambda^{\frac{p_{0}-2}{p_{0}}} & \left|\frac{u(\cdot, t)-A}{r}\right|^{2} d x+f_{Q_{r, t}^{(\lambda)}}|D u|^{p(\cdot)} d z \\
& \leq c f_{Q_{\varrho}^{(\lambda)}}\left[\lambda^{\frac{p_{0}-2}{p_{0}}}\left|\frac{u-A}{\varrho-r}\right|^{2}+\left|\frac{u-A}{\varrho-r}\right|^{p(\cdot)}+|F|^{p(\cdot)}+1\right] d z
\end{aligned}
$$


where $c=c\left(\nu, L, \gamma_{1}, \gamma_{2}\right)$. From this we immediately conclude the desired Caccioppoli inequality by taking in the first term on the left-hand side the supremum with respect to $t \in \Lambda_{r}^{(\lambda)}$ and letting $t \uparrow \lambda^{\frac{2-p_{0}}{p_{0}}} r^{2}$ in the second one.

\section{Poincaré type inequalities}

Due to the character of the weak formulation of (1.1) a weak solution $u$ does not admit the necessary regularity properties for an immediate application of a Poincaré inequality for maps defined on $\mathbb{R}^{n+1}$. Nevertheless, it is possible to establish certain Poincaré-type inequalities for solutions employing the parabolic system. For the spatial directions it is possible to apply slice-wise the standard Poincaré inequality from $\mathbb{R}^{n}$, while for the time direction it is possible to gain — by testing the system - a certain kind of regularity with respect to time. More precisely, it can be shown that the so called weighted means of $u$ are absolutely continuous, and this when combined with a slice-wise application of the Poincaré inequality from $\mathbb{R}^{n}$ leads to suitable Poincaré-type inequalities for weak solutions. Since all estimates have to take into account the non-homogeneous character of the parabolic system we shall establish the Poincaré-type inequalities on intrinsic cylinders $Q_{\varrho}^{(\lambda)}\left(z_{0}\right)$. We start with the following version:

Lemma 5.1. Suppose that $u$ is a weak solution of (1.1) under the assumptions (2.1) and let $F \in L^{p(\cdot)}\left(\Omega_{T}, \mathbb{R}^{N n}\right)$. Furhtermore, let $Q_{\varrho}^{(\lambda)}\left(z_{0}\right) \subseteq$ $\Omega_{T}$ be a parabolic cylinder with $0<\varrho \leq 1$ and $\lambda>0$. Then, for all $\vartheta \in\left[1, p_{1}\right]$ there holds

$$
\begin{array}{r}
f_{Q_{\varrho}^{(\lambda)}\left(z_{0}\right)}\left|\frac{u-(u)_{z_{0} ; \varrho}^{(\lambda)}}{\varrho}\right|^{\vartheta} d z \leq c f_{Q_{\varrho}^{(\lambda)}\left(z_{0}\right)}|D u|^{\vartheta} d z \\
+c\left(\lambda^{\frac{2-p_{0}}{p_{0}}} f_{Q_{\varrho}^{(\lambda)}\left(z_{0}\right)}(|D u|+|F|+1)^{p(\cdot)-1} d z\right)^{\vartheta}
\end{array}
$$

where $c=c\left(n, N, L, \gamma_{2}\right)$.

Proof: In the following we shall omit again the reference point $z_{0}$ in our notation. Let $\eta \in C_{0}^{\infty}\left(B_{\varrho}\right)$ be a nonnegative weight-function satisfying

$$
\eta \geq 0, \quad f_{B_{\varrho}\left(x_{0}\right)} \eta d x=1 \quad \text { and } \quad\|\eta\|_{\infty}+\varrho\|D \eta\|_{\infty} \leq c_{\eta} .
$$


Note that we can arrange $\eta$ in such a way that $c_{\eta}=c_{\eta}(n)$. Then, for a.e. $t \in \Lambda_{\varrho}^{(\lambda)}$ we define the weighted mean of $u(\cdot, t)$ on $B_{\varrho}$ by

$$
(u)_{\eta}(t):=f_{B_{\varrho}} u(\cdot, t) \eta d x .
$$

Having the weighted means of $u(\cdot, t)$ at hand the slice-wise (meaning slice-wise in time) application of the standard Poincaré's inequality with respect to the spatial variable $x$ on $\mathbb{R}^{n}$ is allowed and this will be employed as follows: We first estimate the $\vartheta$-mean deviation of $u$ on $Q_{\varrho}^{(\lambda)}$ (i.e. the deviation of $u$ from its mean on $Q_{\varrho}^{(\lambda)}$ in $L^{\vartheta}$ ) by adding and subtracting the weighted means of $u$ in the following way:

$$
\begin{aligned}
f_{Q_{\varrho}^{(\lambda)}}\left|\frac{u-(u)_{\varrho}^{(\lambda)}}{\varrho}\right|^{\vartheta} d z \leq & 3^{\vartheta-1}\left[f_{Q_{\varrho}^{(\lambda)}}\left|\frac{u-(u)_{\eta}}{\varrho}\right|^{\vartheta} d z\right. \\
& +\varrho^{-\vartheta} f_{\Lambda_{\varrho}^{(\lambda)}}\left|f_{\Lambda_{\varrho}^{(\lambda)}}\left[(u)_{\eta}(t)-(u)_{\eta}(\tau)\right] d \tau\right|^{\vartheta} d t \\
& \left.+\left|\frac{1}{\varrho} f_{\Lambda_{\varrho}^{(\lambda)}}(u)_{\eta}(\tau) d \tau-(u)_{\varrho}^{(\lambda)}\right|^{\vartheta}\right] \\
= & 3^{\vartheta-1}[I+I I+I I I],
\end{aligned}
$$

with the obvious meaning of $I, I I$ and $I I I$. In estimating $I$ we apply the standard Poincaré inequality from $\mathbb{R}^{n}$ "slice-wise" to $u(\cdot, t)$ and infer that

$$
I \leq c(n, \vartheta) f_{Q_{Q}^{(\lambda)}}|D u|^{\vartheta} d z .
$$

For III a similar argument yields

$$
I I I \leq I \leq c(n, \vartheta) f_{Q_{\varrho}^{(\lambda)}}|D u|^{\vartheta} d z .
$$

Since $1 \leq \vartheta \leq p_{1} \leq \gamma_{2}$ and the dependence of the constant $c(n, \vartheta)$ in the Poincaré inequality upon $\vartheta$ is continuous we can replace $c(n, \vartheta)$ by a constant depending only on $n$ and $\gamma_{2}$. 
For the estimate of $I I$ we shall use the parabolic system (1.1) in order to obtain an estimate for the difference $\left|(u)_{\eta}(t)-(u)_{\eta}(\tau)\right|$ of the weighted means for a.e. $t, \tau \in \Lambda_{\varrho}^{(\lambda)}$. The precise argument is as follows: For $i \in\{1, \ldots, N\}$ we choose $\varphi: \mathbb{R}^{n+1} \rightarrow \mathbb{R}^{N}$ with $\varphi_{i}=\eta$ and for the remaining components $\varphi_{j}=0, j \neq i$, as test-function in the Steklovformulation (3.7) of the parabolic system. Then, for $t, \tau \in \Lambda_{\varrho}^{(\lambda)}$ we get

$$
\begin{aligned}
\left(\left[u_{i}\right]_{h}\right)_{\eta}(t)-\left(\left[u_{i}\right]_{h}\right)_{\eta}(\tau) & =\int_{\tau}^{t} \partial_{s}\left(\left[u_{i}\right]_{h}\right)_{\eta} d s \\
& =-\int_{\tau}^{t} f_{B_{\varrho}}\left(\left[a_{i}(\cdot, D u)\right]_{h}+\left[|F|^{p(\cdot)-2} F_{i}\right]_{h}\right) \cdot D \eta d x d s
\end{aligned}
$$

Using the growth condition $(2.1)_{1}$ for the vector field $a(\cdot)$ and the bound $\|D \eta\|_{\infty} \leq c_{\eta} / \varrho$ we find for a.e. $t, \tau \in \Lambda_{\varrho}^{(\lambda)}$, after passing to the limit $h \downarrow 0$, that

$$
\begin{aligned}
\left|\left(u_{i}\right)_{\eta}(t)-\left(u_{i}\right)_{\eta}(\tau)\right| & \leq L \int_{\tau}^{t} f_{B_{\varrho}}\left[(|D u|+1)^{p(\cdot)-1}+|F|^{p(\cdot)-1}\right]|D \eta| d x d s \\
& \leq c \varrho \lambda^{\frac{2-p_{0}}{p_{0}}} f_{Q_{\varrho}^{(\lambda)}}(|D u|+1)^{p(\cdot)-1}+|F|^{p(\cdot)-1} d z
\end{aligned}
$$

for a constant $c=c\left(L, c_{\eta}\right)=c(n, L)$. Summing over $i=1, \ldots, N$ we infer the following estimate for $I I$ :

$$
I I \leq c\left(n, N, L, \gamma_{2},\right)\left(\lambda^{\frac{2-p_{0}}{p_{0}}} f_{Q_{\varrho}^{(\lambda)}}(|D u|+|F|+1)^{p(\cdot)-1} d z\right)^{\vartheta}
$$

Joining the estimates obtained for $I-I I I$ with (5.1) we obtain the desired Poincaré-type inequality.

The previous Poincaré-type inequality has — due to the second integral of the right-hand side - a non-homogeneous character, meaning that $\left(\lambda^{\frac{2-p_{0}}{p_{0}}} f_{Q_{\varrho}^{(\lambda)}}(|D u|+|F|+1)^{p(\cdot)-1} d z\right)^{\vartheta}$ has the wrong exponent $p(\cdot)-1$. In the following corollary we compensate this by introducing a specific intrinsic scaling of the cylinders, which will depend on the solution itself. 
Corollary 5.2. Let $\kappa \geq 1$ and $M>1$ be fixed. Then, there exist $\varrho_{0}=\varrho_{0}\left(n, \gamma_{1}, \kappa, M\right)>0$ and $c=c\left(n, N, L, L_{1}, \gamma_{1}, \gamma_{2}, \kappa\right)$ such that the following holds: Suppose that $u$ is a weak solution of (1.1) under the assumptions (2.1), (2.2), (2.4), satisfying the energy bound (2.7) for the given number $M$, and let $F \in L^{p(\cdot)}\left(\Omega_{T}, \mathbb{R}^{N n}\right)$. Furthermore, assume that for some parabolic cylinder $Q_{\varrho}^{(\lambda)}\left(z_{0}\right) \subseteq \Omega_{T}$ with $0<\varrho \leq \varrho_{0}$ and $\lambda \geq 1$ the following intrinsic coupling holds:

$$
\kappa^{-1} \lambda \leq f_{Q_{\varrho}^{(\lambda)}\left(z_{0}\right)}(|D u|+|F|+1)^{p(\cdot)} d z \leq \kappa \lambda .
$$

Then, for all $\vartheta \in\left[1, p_{1}\right]$ we have

$$
f_{Q_{\varrho}^{(\lambda)}\left(z_{0}\right)}\left|\frac{u-(u)_{z_{0} ; \varrho}^{(\lambda)}}{\varrho}\right|^{\vartheta} d z \leq c \lambda^{\frac{\vartheta}{p_{0}}} .
$$

Proof: Again we omit the reference point $z_{0}$ in our notation. First, we apply the Poincaré-type inequality from Lemma 5.1 and obtain

$$
\begin{aligned}
f_{Q_{\varrho}^{(\lambda)}}\left|\frac{u-(u)_{\varrho}^{(\lambda)}}{\varrho}\right|^{\vartheta} d z \leq c f_{Q_{\varrho}^{(\lambda)}}|D u|^{\vartheta} d z \\
+c\left(\lambda^{\frac{2-p_{0}}{p_{0}}} f_{Q_{\varrho}^{(\lambda)}}(|D u|+|F|+1)^{p(\cdot)-1} d z\right)^{\vartheta}
\end{aligned}
$$

for a constant $c=c\left(n, N, L, \gamma_{2}\right)$. In the following, by $\beta_{n} \equiv \beta_{n}(n)$ we denote the constant from (3.2) and by $\alpha \equiv \alpha\left(n, \gamma_{1}\right) \leq 1$ the exponent defined in (3.3). Then, we fix the radius $\varrho_{0}=\varrho_{0}\left(n, \gamma_{1}, \kappa, M\right)>0$ in such a way that

$$
\varrho_{0} \leq\left(4 \sqrt{\beta_{n} \kappa M}\right)^{-\frac{2}{\alpha}}
$$

This choice allows us to use (3.6) in the sequel. To estimate the first term on the right-hand side of (5.3) we use Hölder's inequality (note that $\vartheta \leq$ $\left.p_{1}\right)$, hypothesis (5.2), the fact that $\kappa, \lambda \geq 1$ and (3.6). Proceeding in this 
way we find

$$
\begin{aligned}
f_{Q_{\varrho}^{(\lambda)}}|D u|^{\vartheta} d z & \leq\left[f_{Q_{\varrho}^{(\lambda)}}|D u|^{p_{1}} d z\right]^{\frac{\vartheta}{p_{1}}} \\
& \leq\left[f_{Q_{\varrho}^{(\lambda)}}(|D u|+1)^{p(\cdot)} d z\right]^{\frac{\vartheta}{p_{1}}} \leq \kappa^{\frac{\vartheta}{p_{1}}} \lambda^{\frac{\vartheta}{p_{1}}} \\
& \leq \kappa \lambda^{\frac{\vartheta\left(p_{0}-p_{1}\right)}{p_{1} p_{0}}} \lambda^{\frac{\vartheta}{p_{0}}} \leq \kappa \lambda^{\frac{p_{2}-p_{1}}{p_{0}}} \lambda^{\frac{\vartheta}{p_{0}}} \\
& \leq \kappa \mathrm{e}^{\frac{4 n L_{1}}{\alpha}} \lambda^{\frac{\vartheta}{p_{0}}}=c\left(n, L_{1}, \gamma_{1}, \kappa\right) \lambda^{\frac{\vartheta}{p_{0}}}
\end{aligned}
$$

To estimate the second term on the right-hand side of (5.3) we first recall that $\omega(\cdot) \leq 1$ and therefore by $(3.3)$ we have $p_{2}-p_{1} \leq 1$ or equivalently $\frac{p_{1}}{p_{2}-1} \geq 1$. Using Hölder's inequality, hypothesis $(5.2), \kappa, \lambda \geq 1, \vartheta \leq p_{1}$ and (3.6) we find

$$
\begin{aligned}
\left(\lambda^{\frac{2-p_{0}}{p_{0}}} f_{Q_{\varrho}^{(\lambda)}}\right. & \left.(|D u|+|F|+1)^{p(\cdot)-1} d z\right)^{\vartheta} \\
& \leq\left(\lambda^{\frac{2-p_{0}}{p_{0}}} f_{Q_{\varrho}^{(\lambda)}}(|D u|+|F|+1)^{p_{2}-1} d z\right)^{\vartheta} \\
& \leq\left[\lambda^{\frac{2-p_{0}}{p_{0}}}\left(f_{Q_{\varrho}^{(\lambda)}}(|D u|+|F|+1)^{p_{1}} d z\right)^{\frac{p_{2}-1}{p_{1}}}\right] \\
& \leq\left[\lambda^{\frac{2-p_{0}}{p_{0}}}\left(f_{Q_{\varrho}^{(\lambda)}}(|D u|+|F|+1)^{p(\cdot)} d z\right)^{\frac{p_{2}-1}{p_{1}}}\right]^{\vartheta} \\
& \leq \kappa^{\frac{\vartheta\left(p_{2}-1\right)}{p_{1}}}\left[\lambda^{\frac{2-p_{0}}{p_{0}}} \lambda^{\frac{p_{2}-1}{p_{1}}}\right]^{\vartheta} \\
& =\kappa^{\frac{\vartheta\left(p_{2}-1\right)}{p_{1}}} \lambda^{-\frac{\vartheta\left(p_{0}-p_{1}\right)}{p_{1} p_{0}}} \lambda^{\frac{\vartheta\left(p_{2}-p_{1}\right)}{p_{1}}} \lambda^{\frac{\vartheta}{p_{0}}} \\
& \leq \kappa^{\gamma_{2} \lambda^{p_{2}-p_{1}}} \lambda^{\frac{\vartheta}{p_{0}}} \leq \kappa^{\gamma_{2}} \mathrm{e}^{\frac{4 n \gamma_{2} L_{1}}{\alpha}} \lambda^{\frac{\vartheta}{p_{0}}}=c \lambda^{\frac{\vartheta}{p_{0}}}
\end{aligned}
$$

where $c=c\left(n, L_{1}, \gamma_{1}, \gamma_{2}, \kappa\right)$. Joining the previous estimates with (5.3) we infer the desired homogeneous Poincaré-type inequality. 
The next lemma can be viewed as an upgrade of Corollary 5.2 with respect to the integrability level. In this sense it can be interpreted as a kind of Sobolev-inequality on intrinsic parabolic cylinders.

Lemma 5.3. Let $\kappa \geq 1$ and $M>1$ be fixed. Then, there exist $\varrho_{0}=$ $\varrho_{0}\left(n, L_{1}, \gamma_{1}, \kappa, M\right)>0$ and $c=c\left(n, N, \nu, L, L_{1}, \gamma_{1}, \gamma_{2}, \kappa\right)$ such that the following holds: Suppose that $u$ is a weak solution of (1.1) where the structure conditions $(2.1),(2.2)$ and $(2.4)$ are in force and $F \in L^{p(\cdot)}\left(\Omega_{T}, \mathbb{R}^{N n}\right)$. Moreover, assume that $u$ satisfies the energy bound from (2.7) for the given number $M$. Finally, suppose that on some parabolic cylinder $Q_{2 \varrho}^{(\lambda)}\left(z_{0}\right) \subseteq \Omega_{T}$ with $0<\varrho \leq \varrho_{0}$ and $\lambda \geq 1$ the following intrinsic coupling holds true:

$$
\kappa^{-1} \lambda \leq f_{Q_{2 \varrho}^{(\lambda)}\left(z_{0}\right)}(|D u|+|F|+1)^{p(\cdot)} d z \leq \kappa \lambda .
$$

Then, with $\sigma \equiv \max \left\{2, p_{2}\right\}$ and $p_{2} \equiv \sup _{Q_{2 \varrho}^{(\lambda)}\left(z_{0}\right)} p(\cdot)$ there holds:

$$
f_{Q_{\varrho}^{(\lambda)}\left(z_{0}\right)}\left|\frac{u-(u)_{z_{0} ; \varrho}^{(\lambda)}}{\varrho}\right|^{\sigma} d z \leq c \lambda^{\frac{\sigma}{p_{0}}} .
$$

Proof: In the following we shall as before omit the reference point $z_{0}$ in our notation. We denote $p_{1} \equiv \inf _{Q_{2 \varrho}^{(\lambda)}} p(\cdot)$. Moreover, we let $\beta_{n} \equiv \beta_{n}(n)$ be the constant from (3.2) and denote by $\alpha \equiv \alpha\left(n, \gamma_{1}\right) \leq 1$ the exponent from (3.3). Then, we fix the radius $\varrho_{0}=\varrho_{0}\left(n, L_{1}, \gamma_{1}, \kappa, M\right)>0$ small enough to have

$$
2 \varrho_{0} \leq\left(4 \sqrt{\beta_{n} \kappa M}\right)^{-\frac{2}{\alpha}} \text { and } \omega\left(8 \sqrt{\beta_{n} \kappa M} \varrho_{0}^{\alpha}\right) \leq \frac{2}{n} .
$$

Note that $(5.6)_{2}$ can always be achieved via $(2.5)$, since $\omega(s) \leq L_{1} \log ^{-1}\left(\frac{1}{s}\right)$, and this yields the dependencies of $\varrho_{0}$ on the indicated parameters. Furthermore, $(5.6)_{1}$ is exactly the radius bound in $(3.4)$ on $Q_{2 \varrho}^{(\lambda)}\left(z_{0}\right)$ and therefore we are allowed to apply $(3.6)$ on $Q_{2 \varrho}^{(\lambda)}$, of course with $p_{1}, p_{2}$ the infimum, respectively supremum of $p(\cdot)$ on $Q_{2 \varrho}^{(\lambda)}$. Finally, $(5.6)_{2}$ and (3.3) yield that

$$
\begin{aligned}
\frac{p_{2}}{p_{1}} & =1+\frac{p_{2}-p_{1}}{p_{1}} \leq 1+p_{2}-p_{1} \leq 1+\omega\left(4 \sqrt{\beta_{n} \kappa M}(2 \varrho)^{\alpha}\right) \\
& \leq 1+\omega\left(8 \sqrt{\beta_{n} \kappa M} \varrho_{0}^{\alpha}\right) \leq \frac{n+2}{n} .
\end{aligned}
$$

Next, we choose $\varrho \leq r<s \leq 2 \varrho$ and apply Gagliardo-Nirenberg's inequality, i.e. Lemma 3.2 with $(\sigma, q, r, \vartheta)$ replaced by $\left(\sigma, p_{1}, 2, \frac{p_{1}}{\sigma}\right)$ "slicewise" to $\left(u-(u)_{r}^{(\lambda)}\right)(\cdot, t)$. The restriction on the parameters necessary 
for the application of the Gagliardo-Nirenberg's inequality can be easily verified using $\frac{p_{2}}{p_{1}} \leq \frac{n+2}{n}$ when $p_{2} \geq 2$, while in the case $p_{2}<2$ it follows from the lower bound $p_{1}>\frac{2 n}{n+2}$. In this way we obtain:

$$
\begin{aligned}
f_{Q_{r}^{(\lambda)}}\left|\frac{u-(u)_{r}^{(\lambda)}}{r}\right|^{\sigma} d z \leq & c f_{\Lambda_{r}^{(\lambda)}} f_{B_{r}}\left|\frac{u-(u)_{r}^{(\lambda)}}{r}\right|^{p_{1}} \\
& +|D u|^{p_{1}} d x\left[f_{B_{r}}\left|\frac{u-(u)_{r}^{(\lambda)}}{r}\right|^{2} d x\right]^{\frac{\sigma-p_{1}}{2}} d t \\
\leq & c f_{Q_{r}^{(\lambda)}}\left|\frac{u-(u)_{r}^{(\lambda)}}{r}\right|^{p_{1}} \\
& +|D u|^{p_{1}} d z\left[\sup _{t \in \Lambda_{r}^{(\lambda)}} f_{B_{r}}\left|\frac{u(\cdot, t)-(u)_{r}^{(\lambda)}}{r}\right|^{2} d x\right]^{\frac{\sigma-p_{1}}{2}}
\end{aligned}
$$

where $c=c(n, \sigma)$. We note that we can replace $\sigma$ by $\gamma_{2}$ in the Gagliardo Nirenberg constant, as the constant depends continuously on the parameter $\sigma \in\left[2, \gamma_{2}\right]$. Next, (5.5) guarantees that the assumption (5.2) of Corollary 5.2 is fulfilled on $Q_{2 \varrho}^{(\lambda)}$. Moreover, the restriction $(5.6)_{1}$ on $\varrho_{0}$ implies the radius restriction $(5.4)$ on $Q_{2 \varrho}^{(\lambda)}$. Therefore, replacing $(u)_{r}^{(\lambda)}$ by $(u)_{2 \varrho}^{(\lambda)}$ via

$$
\left|(u)_{r}^{(\lambda)}-(u)_{2 \varrho}^{(\lambda)}\right|^{\theta} \leq f_{Q_{r}^{(\lambda)}}\left|u-(u)_{2 \varrho}^{(\lambda)}\right|^{\theta} d z \leq 2^{n+2} f_{Q_{2 \varrho}^{(\lambda)}}\left|u-(u)_{2 \varrho}^{(\lambda)}\right|^{\theta} d z
$$

with $\theta \equiv p_{1}$, enlarging the integrability exponent of $|D u|$, applying Corollary 5.2 (for the choice $\vartheta=p_{1}$ ) and using (5.5) and (3.6) we obtain

$$
\begin{aligned}
f_{Q_{r}^{(\lambda)}}\left|\frac{u-(u)_{r}^{(\lambda)}}{r}\right|^{p_{1}}+|D u|^{p_{1}} d z & \leq c\left(n, \gamma_{2}\right) f_{Q_{2 \varrho}^{(\lambda)}}\left|\frac{u-(u)_{2 \varrho}^{(\lambda)}}{2 \varrho}\right|^{p_{1}}+(|D u|+1)^{p(\cdot)} d z \\
& \leq c \lambda^{\frac{p_{1}}{p_{0}}}+c \lambda \leq c \lambda^{\frac{p_{1}}{p_{0}}},
\end{aligned}
$$

where $c \equiv c\left(n, N, L, L_{1}, \gamma_{1}, \gamma_{2}, \kappa\right)$. Inserting this above we arrive at

(5.8) $f_{Q_{r}^{(\lambda)}}\left|\frac{u-(u)_{r}^{(\lambda)}}{r}\right|^{\sigma} d z \leq c \lambda^{\frac{p_{1}}{p_{0}}}\left[\sup _{t \in \Lambda_{r}^{(\lambda)}} f_{B_{r}}\left|\frac{u(\cdot, t)-(u)_{r}^{(\lambda)}}{r}\right|^{2} d x\right]^{\frac{\sigma-p_{1}}{2}}$ 
where $c$ depends as above on $n, N, L, L_{1}, \gamma_{1}, \gamma_{2}$ and $\kappa$. To proceed further we analyze the sup-term in (5.8) with the help of Caccioppoli's inequality from Lemma 4.1 and (5.5). Taking also into account that $\lambda \geq$ 1 we arrive at

$$
\begin{aligned}
& f_{Q_{r}^{(\lambda)}}\left|\frac{u-(u)_{r}^{(\lambda)}}{r}\right|^{\sigma} d z \\
& \leq c \lambda^{\frac{p_{1}}{p_{0}}}\left[f_{Q_{s}^{(\lambda)}}\left|\frac{u-(u)_{r}^{(\lambda)}}{s-r}\right|^{2}+\lambda^{\frac{2-p_{0}}{p_{0}}}\left|\frac{u-(u)_{r}^{(\lambda)}}{s-r}\right|^{p(\cdot)}+\lambda^{\frac{2-p_{0}}{p_{0}}}(|F|+1)^{p(\cdot)} d z\right]^{\frac{\sigma-p_{1}}{2}} \\
& \leq c \lambda^{\frac{p_{1}}{p_{0}}}\left[f_{Q_{s}^{(\lambda)}}\left|\frac{u-(u)_{r}^{(\lambda)}}{s-r}\right|^{2}+\lambda^{\frac{2-p_{0}}{p_{0}}}\left|\frac{u-(u)_{r}^{(\lambda)}}{s-r}\right|^{p_{2}}+\lambda^{\frac{2}{p_{0}}} d z\right]^{\frac{\sigma-p_{1}}{2}},
\end{aligned}
$$

for a constant $c=c\left(n, N, \nu, L, L_{1}, \gamma_{1}, \gamma_{2}, \kappa\right)$. Next, we replace $(u)_{r}^{(\lambda)}$ by $(u)_{s}^{(\lambda)}$ on the right-hand side via the argument from (5.7), apply Hölder's and Young's inequality (note that the occurring exponents are less than or equal to 1 due to our choice of $\varrho_{0}$ ) and finally use (3.6). In this way we find that

$$
\begin{aligned}
f_{Q_{r}^{(\lambda)}} & \left.\frac{u-(u)_{r}^{(\lambda)}}{r}\right|^{\sigma} d z \\
\leq & c \lambda^{\frac{p_{1}}{p_{0}}}\left(f_{Q_{s}^{(\lambda)}}\left|\frac{u-(u)_{s}^{(\lambda)}}{s-r}\right|^{\sigma} d z\right)^{\frac{\sigma-p_{1}}{\sigma}} \\
& +c \lambda^{\frac{2 \sigma-p_{0}\left(\sigma-p_{1}\right)}{2 p_{0}}}\left(f_{Q_{s}^{(\lambda)}}\left|\frac{u-(u)_{s}^{(\lambda)}}{s-r}\right|^{\sigma} d z\right)^{\frac{p_{2}\left(\sigma-p_{1}\right)}{2 \sigma}}+c \lambda^{\frac{\sigma}{p_{0}}} \\
\leq & \frac{1}{2} f_{Q_{s}^{(\lambda)}}\left|\frac{u-(u)_{s}^{(\lambda)}}{s}\right|^{\sigma} d z \\
& +c\left[\left(\frac{s}{s-r}\right)^{\frac{\sigma\left(\sigma-p_{1}\right)}{p_{1}}} \lambda^{\frac{\sigma}{p_{0}}}+\left(\frac{s}{s-r}\right)^{\frac{\sigma p_{2}\left(\sigma-p_{1}\right)}{2 \sigma-p_{2}\left(\sigma-p_{1}\right)}} \lambda^{\frac{\sigma}{p_{0}}+\frac{\sigma\left(p_{2}-p_{0}\right)\left(\sigma-p_{1}\right)}{p_{0}\left[2 \sigma-p_{2}\left(\sigma-p_{1}\right)\right]}}+\lambda^{\frac{\sigma}{p_{0}}}\right] \\
\leq & \frac{1}{2} f_{Q_{s}^{(\lambda)}}\left|\frac{u-(u)_{s}^{(\lambda)}}{s}\right|^{\sigma} d z+c\left(\frac{2 \varrho}{s-r}\right)^{\beta} \lambda^{\frac{\sigma}{p_{0}}},
\end{aligned}
$$


where $\beta \equiv \max \left\{\frac{\sigma\left(\sigma-p_{1}\right)}{p_{1}}, \frac{\sigma p_{2}\left(\sigma-p_{1}\right)}{2 \sigma-p_{2}\left(\sigma-p_{1}\right)}\right\}$ and $c=c\left(n, N, \nu, L, L_{1}, \gamma_{1}, \gamma_{2}, \kappa\right)$. Applying Lemma 3.1 with the choices $\vartheta=\frac{1}{2}, A=c(2 \varrho)^{\beta} \lambda^{\frac{\sigma}{p_{0}}}, C=0$ and

$$
\phi(s)=f_{Q_{s}^{(\lambda)}}\left|\frac{u-(u)_{s}^{(\lambda)}}{s}\right|^{\sigma} d z
$$

we obtain the desired Sobolev-type estimate.

\section{Reverse-Hölder type inequality}

The following lemma is an essential tool in proving the main result, i.e. the higher integrability of the spatial gradients. It roughly states that on intrinsic parabolic cylinders the spatial gradient of a weak solution satisfies a reverse Hölder-inequality in the sense that the $p(\cdot)$-energy of $u$ on $Q_{\varrho}^{(\lambda)}\left(z_{0}\right)$ is controlled by the $p(\cdot) / \vartheta$-energy over $Q_{2 \varrho}^{(\lambda)}\left(z_{0}\right)$ plus some extra term taking into account the inhomogeneity $F$. The reverse Höldertype inequality has exactly the structure from the case $p(\cdot) \equiv$ const; see [29] for the case of parabolic systems of $p$-Laplacean type, or $[7]$ for the corresponding case of higher order, degenerate resp. singular parabolic systems.

Lemma 6.1. Let $M>1$. Then there exist a radius $\varrho_{0}=\varrho_{0}\left(n, L_{1}, \gamma_{1}, \gamma_{2}, M\right)>0$ and a constant $c \equiv c\left(n, N, \nu, L, L_{1}, \gamma_{1}, \gamma_{2}\right)$ such that the following holds: Suppose that $u$ is a weak solution of (1.1) where the structure conditions (2.1), (2.2) and (2.4) are in force and with $F \in L^{p(\cdot)}\left(\Omega_{T}, \mathbb{R}^{N n}\right)$. Moreover, assume that $u$ fulfills the energy bound (2.7) for the given number $M$. Then, on any parabolic cylinder $Q_{8 \varrho}^{(\lambda)}\left(z_{0}\right) \subseteq \Omega_{T}$ with $0<\varrho \leq \varrho_{0}$ and $\lambda \geq 1$ on which an intrinsic coupling is given, in the sense that on $Q_{\varrho}^{(\lambda)}\left(z_{0}\right)$ the lower bound

$$
\lambda \leq f_{Q_{\varrho}^{(\lambda)}\left(z_{0}\right)}(|D u|+|F|+1)^{p(\cdot)} d z
$$

holds, while on $Q_{8 \varrho}^{(\lambda)}\left(z_{0}\right)$ the upper bound

$$
f_{Q_{8 \varrho}^{(\lambda)}\left(z_{0}\right)}(|D u|+|F|+1)^{p(\cdot)} d z \leq \lambda
$$


is fulfilled, there holds the following reverse Hölder inequality

$$
\begin{aligned}
f_{Q_{\varrho}^{(\lambda)}\left(z_{0}\right)}|D u|^{p(\cdot)} d z \leq c\left(f_{Q_{2 \varrho}^{(\lambda)}\left(z_{0}\right)}|D u|^{\frac{p(\cdot)}{\vartheta}} d z\right)^{\vartheta} & \\
& +c f_{Q_{2 \varrho}^{(\lambda)}\left(z_{0}\right)}(|F|+1)^{p(\cdot)} d z,
\end{aligned}
$$

where

$$
\vartheta \equiv \min \left\{\frac{\gamma_{1}(n+2)}{2 n}, \sqrt{\frac{n+2}{n}}, \frac{2 \gamma_{2}}{2 \gamma_{2}-1}\right\}>1 .
$$

Proof: Throughout the proof we shall omit again the reference point $z_{0}$ in our notation. From the Caccioppoli inequality, i.e. Lemma 4.1, we obtain

$$
\begin{aligned}
& f_{Q_{\varrho}^{(\lambda)}}|D u|^{p(\cdot)} d z \leq c f_{Q_{2 \varrho}^{(\lambda)}}\left[\lambda^{\frac{p_{0}-2}{p_{0}}}\left|\frac{u-(u)_{2 \varrho}^{(\lambda)}}{\varrho}\right|^{2}\right. \\
& \left.+\left|\frac{u-(u)_{2 \varrho}^{(\lambda)}}{\varrho}\right|^{p(\cdot)}+|F|^{p(\cdot)}+1\right] d z \\
& \leq c\left(\nu, L, \gamma_{1}, \gamma_{2}\right)\left[\lambda^{\frac{p_{0}-2}{p_{0}}} I_{2}+I_{p_{2}}+1+f_{Q_{2 \varrho}^{(\lambda)}}|F|^{p(\cdot)} d z\right],
\end{aligned}
$$

where, for $\sigma=2$ or $\sigma=p_{2}$, we have abbreviated

$$
I_{\sigma} \equiv f_{Q_{2 \varrho}^{(\lambda)}}\left|\frac{u-(u)_{2 \varrho}^{(\lambda)}}{\varrho}\right|^{\sigma} d z
$$

The exponents $p_{1}$ and $p_{2}$ are taken on $Q_{4 \varrho}^{(\lambda)}\left(z_{0}\right)$, i.e. we have

$$
p_{1}:=\inf _{Q_{4 \varrho}^{(\lambda)}\left(z_{0}\right)} p(\cdot) \text { and } p_{2}:=\sup _{Q_{4 \varrho}^{(\lambda)}\left(z_{0}\right)} p(\cdot) .
$$

Our goal now is to estimate $I_{\sigma}$ for $\sigma=2$ and $\sigma=p_{2}$ in terms of the right-hand side of (6.3). We start by defining

$$
q:=\max \left\{\frac{2 n}{n+2}, \frac{n p_{2}}{n+2}, p_{2}-1\right\} .
$$

Reducing the value of $\varrho_{0}$ eventually we can ensure that

$$
q \leq \frac{p_{1}}{\vartheta}
$$


where $\vartheta$ is defined in (6.4). Indeed, this can be seen as follows: First, we choose the radius $\varrho_{0}=\varrho_{0}\left(n, L_{1}, \gamma_{1}, \gamma_{2}, M\right)>0$ small enough to have:

$$
\omega\left(16 \beta_{n} \sqrt{M} \varrho_{0}^{\alpha}\right) \leq \min \left\{\sqrt{\frac{n+2}{n}}-1, \frac{\gamma_{1}}{2 \gamma_{2}}\right\} .
$$

This is possible thanks to (2.5). Remember that the exponent $\alpha$ has been defined in (3.3), while $\beta_{n}$ is from (3.2). In the following we separately consider the different possibilities for the maximum in (6.6). When $q=$ $\frac{2 n}{n+2}$ we have

$$
\frac{2 n}{n+2} \leq p_{1} \cdot \frac{2 n}{\gamma_{1}(n+2)} \leq \frac{p_{1}}{\vartheta}
$$

while in the case $q=\frac{n p_{2}}{n+2}$ we use (3.3) (with $\kappa=1$ ) to infer

$$
\begin{aligned}
\frac{n p_{2}}{n+2} & =\frac{n p_{1}}{n+2}\left[1+\frac{p_{2}-p_{1}}{p_{1}}\right] \leq \frac{n p_{1}}{n+2}\left[1+\omega\left(4 \beta_{n} \sqrt{M}(4 \varrho)^{\alpha}\right)\right] \\
& \leq \frac{n p_{1}}{n+2}\left[1+\omega\left(16 \beta_{n} \sqrt{M} \varrho_{0}^{\alpha}\right)\right] \leq p_{1} \sqrt{\frac{n}{n+2}} \leq \frac{p_{1}}{\vartheta} .
\end{aligned}
$$

In the remaining case $q=p_{2}-1$ we once again use (3.3) to find that

$$
\begin{aligned}
p_{2}-1 & =p_{1}\left[\frac{p_{1}-1}{p_{1}}+\frac{p_{2}-p_{1}}{p_{1}}\right] \leq p_{1}\left[\frac{\gamma_{2}-1}{\gamma_{2}}+\frac{\omega\left(16 \beta_{n} \sqrt{M} \varrho_{0}^{\alpha}\right)}{\gamma_{1}}\right] \\
& \leq p_{1}\left[\frac{\gamma_{2}-1}{\gamma_{2}}+\frac{1}{2 \gamma_{2}}\right]=p_{1} \cdot \frac{2 \gamma_{2}-1}{2 \gamma_{2}} \leq \frac{p_{1}}{\vartheta} .
\end{aligned}
$$

Altogether this shows that (6.7) holds.

Now from (6.7) we trivially have $q<p_{1}$ and therefore $|D u| \in L^{q}\left(Q_{2 \varrho}^{(\lambda)}\right)$. Hence, we can apply Gagliardo-Nirenberg's inequality -i.e. Lemma 3.2 with $(\sigma, q, r, \vartheta)$ replaced by $\left(\sigma, q, 2, \frac{q}{\sigma}\right)$ — "slice-wise" to $\left(u-(u)_{2 \varrho}^{(\lambda)}\right)(\cdot, t)$. The restriction on the parameters is fulfilled due to the particular choice of $q$ in (6.6) (especially the first two entries from the maximum construction). We infer

$$
\begin{aligned}
I_{\sigma} & \leq c f_{\Lambda_{2 \varrho}^{(\lambda)}} f_{B_{2 \varrho}}\left|\frac{u-(u)_{2 \varrho}^{(\lambda)}}{\varrho}\right|^{q}+|D u|^{q} d x\left[f_{B_{2 \varrho}}\left|\frac{u-(u)_{2 \varrho}^{(\lambda)}}{\varrho}\right|^{2} d x\right]^{\frac{\sigma-q}{2}} d t \\
& \leq c J^{\frac{\sigma-q}{2}} f_{Q_{2 \varrho}^{(\lambda)}}\left|\frac{u-(u)_{2 \varrho}^{(\lambda)}}{\varrho}\right|^{q}+|D u|^{q} d z
\end{aligned}
$$


where $c=c\left(n, \gamma_{2}\right)$; note that $\sigma \in\left\{2, p_{2}\right\}$ and therefore we can replace the dependence in the Gagliardo-Nirenberg constant upon $\sigma$ by the one on $\gamma_{2}$. In (6.8) we abbreviated:

$$
J \equiv \sup _{t \in \Lambda_{2 \varrho}^{(\lambda)}} f_{B_{2 \varrho}}\left|\frac{u(\cdot, t)-(u)_{2 \varrho}^{(\lambda)}}{\varrho}\right|^{2} d x .
$$

In order to estimate $J$ we apply the Caccioppoli inequality from Lemma 4.1 yielding that

$$
\begin{aligned}
J & \leq c f_{Q_{4 \varrho}^{(\lambda)}}\left|\frac{u-(u)_{2 \varrho}^{(\lambda)}}{\varrho}\right|^{2}+\lambda^{\frac{2-p_{0}}{p_{0}}}\left|\frac{u-(u)_{2 \varrho}^{(\lambda)}}{\varrho}\right|^{p(\cdot)}+\lambda^{\frac{2-p_{0}}{p_{0}}}(|F|+1)^{p(\cdot)} d z \\
& \leq c f_{Q_{4 \varrho}^{(\lambda)}}\left|\frac{u-(u)_{4 \varrho}^{(\lambda)}}{\varrho}\right|^{2}+\lambda^{\frac{2-p_{0}}{p_{0}}}\left|\frac{u-(u)_{4 \varrho}^{(\lambda)}}{\varrho}\right|^{p_{2}}+\lambda^{\frac{2}{p_{0}}} d z,
\end{aligned}
$$

for a constant $c=c\left(\nu, L, \gamma_{1}, \gamma_{2}\right)$. Here we have used (6.2) and $\lambda \geq 1$ in the last line. Moreover, as in in the proof of Lemma 5.3 (see (5.7) there) we replaced $(u)_{2 \varrho}^{(\lambda)}$ by $(u)_{4 \varrho}^{(\lambda)}$.

Next, reducing eventually the value of $\varrho_{0}$ - without changing the dependencies upon the various parameters - we may apply Lemma 5.3 with $\left(4 \varrho, 8^{n+2}\right)$ instead of $(\varrho, \kappa)$. Note that this further restriction on the size of $\varrho_{0}$ (see $\left.(5.6)_{1}\right)$ also ensures the validity of (3.6). At this stage we apply Lemma 5.3 with exponents $\sigma=2$ and $\sigma=p_{2}$ on $Q_{4 \varrho}^{(\lambda)}$; this is possible due to the hypothesis (6.1) and (6.2), one only has to replace $\kappa$ by $8^{n+2}$ in the application. We arrive at

$$
J \leq c\left(\lambda^{\frac{2}{p_{0}}}+\lambda^{\frac{2-p_{0}+p_{2}}{p_{0}}}\right) \leq c\left(n, N, \nu, L, L_{1}, \gamma_{1}, \gamma_{2}\right) \lambda^{\frac{2}{p_{0}}} .
$$

Here, we have used (3.6) in order to control $\lambda^{\frac{p_{2}-p_{0}}{p_{0}}}$ by $\mathrm{e}^{\frac{4 n L_{1}}{\alpha}}$. Plugging this into $(6.8)$ we get

$$
I_{\sigma} \leq c \lambda^{\frac{\sigma-q}{p_{0}}} f_{Q_{2 \varrho}^{(\lambda)}}\left|\frac{u-(u)_{2 \varrho}^{(\lambda)}}{\varrho}\right|^{q}+|D u|^{q} d z
$$

where $c=c\left(n, N, \nu, L, L_{1}, \gamma_{1}, \gamma_{2}\right)$. In order to estimate the integral

$$
f_{Q_{2 \varrho}^{(\lambda)}}\left|\frac{u-(u)_{2 \varrho}^{(\lambda)}}{\varrho}\right|^{q} d z
$$


in (6.9) we shall use the Poincaré-type inequality from Lemma 5.1. Recalling that $q \leq p_{1}$ by (6.7) the application is possible and yields

$$
\begin{aligned}
f_{Q_{2 \varrho}^{(\lambda)}}\left|\frac{u-(u)_{\varrho}^{(\lambda)}}{\varrho}\right|^{q} d z \leq c f_{Q_{2 \varrho}^{(\lambda)}}|D u|^{q} d z \\
+c \lambda^{\frac{q\left(2-p_{0}\right)}{p_{0}}}\left(f_{Q_{2 \varrho}^{(\lambda)}}(|D u|+|F|+1)^{p(\cdot)-1} d z\right)^{q}
\end{aligned}
$$

for a constant $c=c\left(n, N, L, \gamma_{2}\right)$. In the second integral on the right-hand side we first enlarge the exponent from $p(\cdot)$ to $p_{2}$ and then apply Hölder's inequality (which is allowed since by (6.7) we have that $p_{2}-1 \leq q \leq \frac{p_{1}}{\vartheta}$ ) as follows:

$$
\begin{aligned}
\left(f_{Q_{2 \varrho}^{(\lambda)}}(|D u|+|F|+1)^{p(\cdot)-1} d z\right)^{q} & \leq\left(f_{Q_{2 \varrho}^{(\lambda)}}(|D u|+|F|+1)^{p_{2}-1} d z\right)^{q} \\
& \leq\left(f_{Q_{2 \varrho}^{(\lambda)}}(|D u|+|F|+1)^{\frac{p_{1}}{\vartheta}} d z\right)^{\frac{\vartheta q\left(p_{2}-1\right)}{p_{1}}} \\
& \leq\left(f_{Q_{2 \varrho}^{(\lambda)}}(|D u|+|F|+1)^{\frac{p(\cdot)}{\vartheta}} d z\right)^{\frac{\vartheta q\left(p_{2}-1\right)}{p_{1}}}
\end{aligned}
$$

Similarly, we obtain

$$
f_{Q_{2 \varrho}^{(\lambda)}}|D u|^{q} d z \leq\left(f_{Q_{2 \varrho}^{(\lambda)}}(|D u|+1)^{\frac{p(\cdot)}{\vartheta}} d z\right)^{\frac{\vartheta q}{p_{1}}} .
$$

Inserting the preceding inequalities into (6.10) and joining the result with (6.9) we arrive at

$$
\begin{aligned}
I_{\sigma} \leq c & {\left[\lambda^{\frac{\sigma-q}{p_{0}}}\left(f_{Q_{2 \varrho}^{(\lambda)}}(|D u|+1)^{\frac{p(\cdot)}{\vartheta}} d z\right)^{\frac{\vartheta q}{p_{1}}}\right.} \\
& \left.+\lambda^{\frac{\sigma-q+q\left(2-p_{0}\right)}{p_{0}}}\left(f_{Q_{2 \varrho}^{(\lambda)}}(|D u|+|F|+1)^{\frac{p(\cdot)}{\vartheta}} d z\right)^{\frac{\vartheta q\left(p_{2}-1\right)}{p_{1}}}\right],
\end{aligned}
$$

for a constant $c=c\left(n, N, \nu, L, L_{1}, \gamma_{1}, \gamma_{2}\right)$. To bound the second integral on the right-hand side of (6.11) we distinguish between the cases $p_{2} \geq 2$ and $p_{2}<2$. When $p_{2} \geq 2$ we apply Hölder's inequality, enlarge the 
domain of integration from $Q_{2 \varrho}^{(\lambda)}$ to $Q_{8 \varrho}^{(\lambda)}$ and then use (6.2). Finally, we recall $\lambda \geq 1$ and utilize (3.6). In this way we obtain:

$$
\begin{aligned}
\lambda^{\frac{q\left(2-p_{0}\right)}{p_{0}}} & \left(f_{Q_{2 \varrho}^{(\lambda)}}(|D u|+|F|+1)^{\frac{p(\cdot)}{\vartheta}} d z\right)^{\frac{\vartheta q\left(p_{2}-2\right)}{p_{1}}} \\
& \leq \lambda^{\frac{q\left(2-p_{0}\right)}{p_{0}}}\left(4^{n+2} f_{Q_{8 \varrho}^{(\lambda)}}(|D u|+|F|+1)^{p(\cdot)} d z\right)^{\frac{q\left(p_{2}-2\right)}{p_{1}}} \\
& \leq c \lambda^{\frac{q\left(p_{2}-p_{1}\right)}{p_{1}}-\frac{2 q\left(p_{0}-p_{1}\right)}{p_{1} p_{0}}} \leq c \lambda^{p_{2}-p_{1}} \\
& \leq c \mathrm{e}^{\frac{4 n \gamma_{2} L_{1}}{\alpha}}=c\left(n, L_{1}, \gamma_{1}, \gamma_{2}\right) .
\end{aligned}
$$

Combining this with (6.11) we find

$$
I_{\sigma} \leq c \lambda^{\frac{\sigma-q}{p_{0}}}\left(f_{Q_{2 \varrho}^{(\lambda)}}(|D u|+|F|+1)^{\frac{p(\cdot)}{\vartheta}} d z\right)^{\frac{\vartheta q q}{p_{1}}},
$$

again for a constant $c=c\left(n, N, \nu, L, L_{1}, \gamma_{1}, \gamma_{2}\right)$. In the case $p_{2}<2$ we use (3.6) and apply Young's inequality to find for $\delta>0$ that there holds:

$$
\begin{aligned}
& \lambda^{\frac{\sigma-q+q\left(2-p_{0}\right)}{p_{0}}}\left(f_{Q_{2 \varrho}^{(\lambda)}}(|D u|+|F|+1)^{\frac{p(\cdot)}{\vartheta}} d z\right)^{\frac{\vartheta q\left(p_{2}-1\right)}{p_{1}}} \\
& \quad=\lambda^{\frac{\sigma\left(2-p_{2}\right)}{p_{0}}} \lambda^{\frac{q\left(p_{2}-p_{0}\right)}{p_{0}}} \lambda^{\frac{(\sigma-q)\left(p_{2}-1\right)}{p_{0}}}\left(f_{Q_{2 \varrho}^{(\lambda)}}(|D u|+|F|+1)^{\frac{p(\cdot)}{\vartheta}} d z\right)^{\frac{\vartheta q\left(p_{2}-1\right)}{p_{1}}} \\
& \leq \lambda^{\frac{\sigma\left(2-p_{2}\right)}{p_{0}}} \mathrm{e}^{\frac{4 n L_{1} \gamma_{2}}{\alpha}} \lambda^{\frac{(\sigma-q)\left(p_{2}-1\right)}{p_{0}}}\left(f_{Q_{2 \varrho}^{(\lambda)}}(|D u|+|F|+1)^{\frac{p(\cdot)}{\vartheta}} d z\right)^{\frac{\vartheta q\left(p_{2}-1\right)}{p_{1}}} \\
& \leq \delta \lambda^{\frac{\sigma}{p_{0}}}+c \delta^{-\frac{2-p_{2}}{p_{2}-1}} \lambda^{\frac{\sigma-q}{p_{0}}}\left(f_{Q_{2 \varrho}^{(\lambda)}}(|D u|+|F|+1)^{\frac{p(\cdot)}{\vartheta}} d z\right)^{\frac{\vartheta q}{p_{1}}}
\end{aligned}
$$

where $c=c\left(n, L_{1}, \gamma_{1}, \gamma_{2}\right)$. Inserting this into (6.11), reducing the value of $\delta$ eventually (i.e. replacing $\delta$ by $\delta / c$ ) and finally noting that $\frac{2-p_{2}}{p_{2}-1} \leq$ 
$\frac{1}{\gamma_{1}-1}$, we obtain

$$
I_{\sigma} \leq \delta \lambda^{\frac{\sigma}{p_{0}}}+c \delta^{-\frac{1}{\gamma_{1}-1}} \lambda^{\frac{\sigma-q}{p_{0}}}\left(f_{Q_{2 \varrho}^{(\lambda)}}(|D u|+|F|+1)^{\frac{p(\cdot)}{\vartheta}} d z\right)^{\frac{\vartheta q}{p_{1}}}
$$

for a constant $c=c\left(n, N, \nu, L, L_{1}, \gamma_{1}, \gamma_{2}\right)$. Altogether we have shown that in any case (6.12) holds. Joining (6.12) for $\sigma=2$ and $\sigma=p_{2}$ with (6.5) and taking again (3.6) into account (in order to have $\lambda^{p_{0}-p_{1}} \leq \mathrm{e}^{\frac{4 n \gamma_{2} L_{1}}{\alpha}}$ and $\left.\lambda^{p_{2}-p_{1}} \leq \mathrm{e}^{\frac{4 n \gamma_{2} L_{1}}{\alpha}}\right)$ we arrive at

$$
\begin{aligned}
f_{Q_{\varrho}^{(\lambda)}}|D u|^{p(\cdot)} d z \leq c \delta^{-\frac{1}{\gamma_{1}-1}} \lambda^{\frac{p_{1}-q}{p_{0}}} & \left(f_{Q_{2 \varrho}^{(\lambda)}}(|D u|+|F|+1)^{\frac{p(\cdot)}{\vartheta}} d z\right)^{\frac{\vartheta q}{p_{1}}} \\
& +c \delta \lambda+c f_{Q_{2 \varrho}^{(\lambda)}}(|F|+1)^{p(\cdot)} d z
\end{aligned}
$$

where $c \equiv c\left(n, N, \nu, L, L_{1}, \gamma_{1}, \gamma_{2}\right)$. We can further estimate the righthand side with Young's inequality; for $\mu>0$ to be chosen later we have:

$$
\begin{aligned}
f_{Q_{\varrho}^{(\lambda)}}|D u|^{p(\cdot)} d z \leq[\mu+c \delta] \lambda+c \delta^{-\frac{\gamma_{2}}{\gamma_{1}-1}} \mu^{-\gamma_{2}} & \left(f_{Q_{2 \varrho}^{(\lambda)}}(|D u|+|F|+1)^{\frac{p(\cdot)}{\vartheta}} d z\right)^{\vartheta} \\
& +c f_{Q_{2 \varrho}^{(\lambda)}}(|F|+1)^{p(\cdot)} d z
\end{aligned}
$$

Here we used $\lambda^{\frac{p_{1}}{p_{0}}} \leq \lambda$ since $\lambda \geq 1$ and $\frac{p_{1}}{q\left(\gamma_{1}-1\right)} \leq \frac{\gamma_{2}}{\gamma_{1}-1}$ and $\frac{p_{1}-q}{q} \leq \gamma_{2}$. At this stage we use (6.1) in order to bound $\lambda^{p_{0}}$ in the following form:

$$
\lambda \leq c\left(\gamma_{2}\right) f_{Q_{\varrho}^{(\lambda)}\left(z_{0}\right)}|D u|^{p(\cdot)} d z+c\left(n, \gamma_{2}\right) f_{Q_{2 \varrho}^{(\lambda)}\left(z_{0}\right)}(|F|+1)^{p(\cdot)} d z .
$$

Using this above and choosing $\mu \equiv \frac{1}{4 c\left(\gamma_{2}\right)}$ and $\delta \equiv \frac{1}{4 c c\left(\gamma_{2}\right)}$ we can reabsorb the integral $\frac{1}{2} f_{Q_{\varrho}^{(\lambda)}\left(z_{0}\right)}|D u|^{p(\cdot)} d z$ into the left-hand side and arrive at

$f_{Q_{\varrho}^{(\lambda)}}|D u|^{p(\cdot)} d z \leq c\left(f_{Q_{2 \varrho}^{(\lambda)}}(|D u|+|F|+1)^{\frac{p(\cdot)}{\vartheta}} d z\right)^{\vartheta}+c f_{Q_{2 \varrho}^{(\lambda)}}(|F|+1)^{p(\cdot)} d z$,

where $c \equiv c\left(n, N, \nu, L, L_{1}, \gamma_{1}, \gamma_{2}\right)$. From this the asserted reverse Hölder inequality (6.3) follows by applying Young's and Hölder's inequality. This finally completes the proof of the lemma. 


\section{Proof of the higher integrability}

As we have seen in the previous section a reverse Hölder inequality is available on intrinsic parabolic cylinders only; see Lemma 6.1. Therefore, the main objective in the proof of the higher integrability theorem is to find parabolic cylinders covering the upper-level set of the spatial gradient in the sense of a Vitali-type covering, such that on each cylinder the intrinsic coupling in the form of (6.1) and (6.2) holds. In order to find such intrinsic cylinders we will use a stopping time argument, which exploits in a certain way the continuous dependence of the integral on the domain of integration. The system of intrinsic cylinders covering the upper-level set of $|D u|$ however does not yield a Vitali-type covering in the usual sense. The peculiarity thereby is that the family of intrinsic cylinders is not uniform - even if the scaling factor $\lambda$ is fixed - in the sense that the scaling of a cylinder around a point $z_{0}$ is $\lambda^{\frac{2-p_{0}}{p_{0}}}$ and hence depends on $p_{0}=p\left(z_{0}\right)$. In order to overcome this technical point we need to have the following version of Vitali's covering theorem for nonuniformly intrinsic parabolic cylinders. To formulate the lemma we recall the definitions of $\beta_{n} \equiv \beta_{n}(n)$ from (3.2) and $\alpha \equiv \alpha\left(n, \gamma_{1}\right)$ from (3.3).

Lemma 7.1. Let $M \geq 1, \lambda \geq 1$ and let $p: \Omega_{T} \rightarrow\left(\gamma_{1}, \gamma_{2}\right)$ fulfill the assumptions (2.2) and (2.4). Then, there exists $\chi \equiv \chi\left(n, L_{1}, \gamma_{1}\right) \geq 5$ such that the following is true: Let $\mathcal{F}=\left\{Q_{i}\right\}_{i \in \mathcal{I}}$ be a family of axially parallel parabolic cylinders of the form

$$
Q_{i} \equiv Q_{\varrho_{i}}^{(\lambda)}\left(z_{i}\right) \equiv B_{\varrho_{i}}\left(x_{i}\right) \times\left(t_{i}-\lambda^{\frac{2-p\left(z_{i}\right)}{p\left(z_{i}\right)}} \varrho_{i}^{2}, t_{i}+\lambda^{\frac{2-p\left(z_{i}\right)}{p\left(z_{i}\right)}} \varrho_{i}^{2}\right)
$$

with uniformly bounded radii, uniformly in the sense that

$$
\varrho_{i} \leq \min \left\{\varrho_{0},\left[\left(\beta_{n} M\right)^{\frac{p\left(z_{i}\right)}{2}} \lambda^{-1}\right]^{\frac{2}{p\left(z_{i}\right)(n+2)}}\right\} \quad \forall i \in \mathcal{I}
$$

where

$$
\varrho_{0}:=\left[6 \sqrt{\beta_{n} M}\right]^{-\frac{2}{\alpha}} .
$$

Then, there exists a countable sub-collection $\mathcal{G} \subseteq \mathcal{F}$ of disjoint parabolic cylinders, such that

$$
\bigcup_{Q \in \mathcal{F}} Q \subset \bigcup_{Q \in \mathcal{G}} \chi Q,
$$

where $\chi Q$ denotes the $\chi$-times enlarged cylinder $Q$, i.e. if $Q \equiv Q_{\varrho}^{(\lambda)}(z)$ then $\chi Q \equiv Q_{\chi \varrho}^{(\lambda)}(z)$.

Proof: We set

$$
R:=\sup \left\{\varrho: Q_{\varrho}^{(\lambda)}(z) \in \mathcal{F}\right\} \leq \varrho_{0} .
$$


Moreover, for $j=1,2, \ldots$ we define

$$
\mathcal{F}_{j}:=\left\{Q_{\varrho}^{(\lambda)}(z) \in \mathcal{F}: \frac{R}{2^{j}}<\varrho \leq \frac{R}{2^{j-1}}\right\}
$$

and choose disjoint subcollections $\mathcal{G}_{j} \subset \mathcal{F}_{j}$ as follows. Let $\mathcal{G}_{1}$ be any maximal disjoint collection of cylinders in $\mathcal{F}_{1}$. Assuming that $\mathcal{G}_{1}, \mathcal{G}_{2}, \ldots, \mathcal{G}_{k-1}$ have been selected, we choose $\mathcal{G}_{k}$ to be any maximal disjoint subcollection of

$$
\left\{Q \in \mathcal{F}_{k}: Q \cap \widehat{Q}=\emptyset \text { for all } \widehat{Q} \in \bigcup_{j=1}^{k-1} \mathcal{G}_{j}\right\} .
$$

Finally, we define

$$
\mathcal{G}:=\bigcup_{j=1}^{\infty} \mathcal{G}_{j}
$$

Obviously $\mathcal{G} \subseteq \mathcal{F}$ is a countable collection of pair-wise disjoint cylinders and it only remains to establish the covering property (7.2). This follows once we can show that for every $Q \in \mathcal{F}$ there exists $\widehat{Q} \in \mathcal{G}$ such that $Q \cap \widehat{Q} \neq \emptyset$ and $Q \subseteq \chi \widehat{Q}$. Let $Q \equiv Q_{\varrho}^{(\lambda)}(z) \in \mathcal{F}$. Then, we find $j \in \mathbb{N}$, such that $Q \in \mathcal{F}_{j}$. Due to the maximality of $\mathcal{G}_{j}$ there exists $\widehat{Q} \equiv Q_{\hat{\varrho}}^{(\lambda)}(\hat{z}) \in \bigcup_{k=1}^{j} \mathcal{G}_{k}$ with $Q \cap \widehat{Q} \neq \emptyset$. From the definition of $\mathcal{F}_{j}$ in (7.3) we infer that $\varrho \leq \frac{R}{2^{j-1}} \leq 2 \hat{\varrho}$. This immediately implies

$$
B_{\varrho}(x) \subseteq B_{5 \varrho}(\hat{x}) \text {. }
$$

The inclusion with respect to the time-direction can be inferred by the following argument: Initially, we have

$$
|t-\hat{t}|+\lambda^{\frac{2-p(z)}{p(z)}} \varrho^{2} \leq 2 \lambda^{\frac{2-p(z)}{p(z)}} \varrho^{2}+\lambda^{\frac{2-p(\hat{z})}{p(\hat{z})}} \hat{\varrho}^{2} .
$$

To proceed further, slightly different arguments are needed to handle the cases $p(z) \geq p(\hat{z})$ and $p(z)<p(\hat{z})$. We start with the case $p(z) \geq p(\hat{z})$. Since $\lambda \geq 1$ and $\varrho \leq 2 \hat{\varrho}$ we conclude that

$$
|t-\hat{t}|+\lambda^{\frac{2-p(z)}{p(z)}} \varrho^{2} \leq 9 \lambda^{\frac{2-p(\hat{z})}{p(\hat{z})}} \hat{\varrho}^{2} .
$$

In the case $p(z)<p(\hat{z})$ we obtain

$$
|t-\hat{t}|+\lambda^{\frac{2-p(z)}{p(z)}} \varrho^{2} \leq 9 \lambda^{\frac{2-p(z)}{p(z)}} \hat{\varrho}^{2}=9 \lambda^{\frac{2-p(\hat{z})}{p(\hat{z})}} \hat{\varrho}^{2} \lambda^{\frac{p(\hat{z})-p(z)}{p(z) p(z)}} .
$$

Here, we still have to bound the factor $\lambda^{\frac{p(z)-p(z)}{p(z) p(z)}}$ by a universal constant depending only on the structural data. For this aim we first shall bound the difference $p(\hat{z})-p(z)$ which can be achieved via (2.2) as follows:

$$
p(\hat{z})-p(z) \leq \omega\left(d_{\mathcal{P}}(\hat{z}, z)\right) \leq \omega\left(\varrho+\hat{\varrho}+\lambda^{\frac{2-p(z)}{2 p(z)}} \varrho+\lambda^{\frac{2-p(\hat{z})}{2 p(\hat{z})}} \varrho\right) .
$$


In the case $p(\hat{z}) \geq 2$ we immediately have $\lambda^{\frac{2-p(\hat{z})}{2 p(\hat{z})}} \hat{\varrho} \leq \hat{\varrho}$ while in the case $\frac{2 n}{n+2}<\gamma_{1} \leq p(\hat{z})<2$ we use the bound (7.1) in the form $\lambda \leq$ $\left[\beta_{n} M \varrho^{-(n+2)}\right]^{\frac{p(\hat{z})}{2}}$ to get

$$
\lambda^{\frac{2-p(\hat{z})}{2 p(z)}} \hat{\varrho} \leq\left[\beta_{n} M \hat{\varrho}^{-(n+2)}\right]^{\frac{2-p(\hat{z})}{4}} \hat{\varrho} \leq \sqrt{\beta_{n} M} \hat{\varrho}^{1-\frac{(n+2)\left(2-\gamma_{1}\right)}{4}} .
$$

Hence, in any case we have

$$
\lambda^{\frac{2-p(\hat{z})}{2 p(z)}} \hat{\varrho} \leq \sqrt{\beta_{n} M} \hat{\varrho}^{1-\frac{(n+2)\left(2-\gamma_{1}\right)}{4}},
$$

and by the same argument applied with $(\hat{\varrho}, \hat{z})$ replaced by $(\varrho, z)$ we also obtain

$$
\lambda^{\frac{2-p(z)}{2 p(z)}} \varrho \leq \sqrt{\beta_{n} M} \varrho^{1-\frac{(n+2)\left(2-\gamma_{1}\right)}{4}} \leq 2 \sqrt{\beta_{n} M} \varrho^{1-\frac{(n+2)\left(2-\gamma_{1}\right)}{4}},
$$

where we used $\varrho \leq 2 \hat{\varrho}$ in the last inequality. Inserting the last two estimates into (7.6) we arrive at

$$
p(\hat{z})-p(z) \leq \omega\left(6 \sqrt{\beta_{n} M} \hat{\varrho}^{1-\frac{(n+2)\left(2-\gamma_{1}\right)}{4}}\right) \leq \omega\left(\Gamma \hat{\varrho}^{\gamma_{1} \frac{n+2}{4}-\frac{n}{2}}\right)
$$

with $\Gamma \equiv 6 \sqrt{\beta_{n} M}$. Note that $\varrho_{0} \equiv \Gamma^{-\frac{2}{\alpha}}$. Taking into account the definition of $\alpha$ from (3.3) we have shown that

$$
p(\hat{z})-p(z) \leq \omega\left(\Gamma \hat{\varrho}^{\alpha}\right) .
$$

This allows us to use (2.5) as follows:

$$
\begin{aligned}
\hat{\varrho}^{-(p(\hat{z})-p(z))} & \leq \hat{\varrho}^{-\omega\left(\Gamma \hat{\varrho}^{\alpha}\right)}=\exp \left[\omega\left(\Gamma \hat{\varrho}^{\alpha}\right) \log \frac{1}{\hat{\varrho}}\right]=\exp \left[\alpha^{-1} \omega\left(\Gamma \hat{\varrho}^{\alpha}\right) \log \frac{1}{\hat{\varrho}^{\alpha}}\right] \\
& =\exp \left[\alpha^{-1} \omega\left(\Gamma \hat{\varrho}^{\alpha}\right)\left(\log \frac{1}{\Gamma \hat{\varrho}^{\alpha}}+\log \Gamma\right)\right] \\
& \leq \mathrm{e}^{\frac{L_{1}}{\alpha}} \exp \left[\alpha^{-1} \omega\left(\Gamma \hat{\varrho}^{\alpha}\right) \log \Gamma\right] \\
& \leq \mathrm{e}^{\frac{L_{1}}{\alpha}} \exp \left[\alpha^{-1} \omega\left(\Gamma \varrho_{0}^{\alpha}\right) \log \Gamma\right] .
\end{aligned}
$$

At this stage we use $\varrho_{0}:=\Gamma^{-\frac{2}{\alpha}}$ and (2.5) to obtain that

$$
\hat{\varrho}^{-(p(\hat{z})-p(z))} \leq \mathrm{e}^{\frac{L_{1}}{\alpha}} \exp \left[\alpha^{-1} \omega\left(\Gamma^{-1}\right) \log \Gamma\right] \leq \mathrm{e}^{\frac{2 L_{1}}{\alpha}} .
$$

Using $\lambda \geq 1$, the bound (7.1) in the form $\lambda \leq\left[\Gamma^{2} \hat{\varrho}^{-(n+2)}\right]^{\frac{p(\hat{z})}{2}}$, the preceding estimate, the definition of $\varrho_{0}$ and (2.5) we find

$$
\begin{aligned}
\lambda^{\frac{p(\hat{z})-p(z)}{p(z) p(z)}} & \leq \lambda^{\frac{p(\hat{z})-p(z)}{p(\hat{z})}} \leq \Gamma^{p(\hat{z})-p(z)} \hat{\varrho}^{-\frac{n+2}{2}(p(\hat{z})-p(z))} \leq \Gamma^{\omega\left(\Gamma \hat{\varrho}^{\alpha}\right)} \mathrm{e}^{\frac{L_{1}(n+2)}{\alpha}} \\
& \leq \Gamma^{\omega\left(\Gamma \varrho_{0}^{\alpha}\right)} \mathrm{e}^{\frac{L_{1}(n+2)}{\alpha}}=\Gamma^{\omega\left(\Gamma^{-1}\right)} \mathrm{e}^{\frac{L_{1}(n+2)}{\alpha}} \leq \mathrm{e}^{\frac{L_{1}(n+2)}{\alpha}+L_{1}} \leq \mathrm{e}^{\frac{4 n L_{1}}{\alpha}}
\end{aligned}
$$


Combining this with (7.5) yields

$$
|t-\hat{t}|+\lambda^{\frac{2-p(z)}{p(z)}} \varrho^{2} \leq 9 \lambda^{\frac{2-p(\hat{z})}{p(\hat{z})}} \hat{\varrho}^{2} \lambda^{\frac{p(\hat{z})-p(z)}{p(\hat{z}) p(z)}} \leq 9 \mathrm{e}^{\frac{4 n L_{1}}{\alpha}} \lambda^{\frac{2-p(\hat{z})}{p(\hat{z})}} \hat{\varrho}^{2} .
$$

Joining this with the corresponding estimate (7.4) from the case $p(z) \geq$ $p(\hat{z})$ we arrive at the final estimate for the time direction:

$$
|t-\hat{t}|+\lambda^{\frac{2-p(z)}{p(z)}} \varrho^{2} \leq \chi^{2} \lambda^{\frac{2-p(\hat{z})}{p(\hat{z})}} \hat{\varrho}^{2},
$$

where we have defined

$$
\chi \equiv 3 \mathrm{e}^{\frac{2 n L_{1}}{\alpha}} \geq 5 .
$$

This implies the inclusion with respect to the time direction

$$
\left(t-\lambda^{\frac{2-p(z)}{p(z)}} \varrho^{2}, t+\lambda^{\frac{2-p(z)}{p(z)}} \varrho^{2}\right) \subseteq\left(\hat{t}-\lambda^{\frac{2-p(\hat{z})}{p(\hat{z})}}(\chi \hat{\varrho})^{2}, \hat{t}+\lambda^{\frac{2-p(\hat{z})}{p(\hat{z})}}(\chi \hat{\varrho})^{2}\right),
$$

which together with the spatial inclusion $B_{\varrho}(x) \subseteq B_{5 \varrho}(\hat{x})$ from above, proves the assertion $Q \subseteq \chi \widehat{Q}$.

Now we have all pre-requisites at hand to give the

Proof of Theorem 2.2: We define

$$
\lambda_{0}^{\frac{1}{d\left(p_{m}\right)}+\frac{2}{p_{M}}-\frac{2}{p_{m}}}:=f_{Q_{2 r}}(|D u|+|F|+1)^{p(\cdot)} d z \geq 1,
$$

where

$$
p_{m}:=\inf _{Q_{2 r}} p(\cdot) \quad \text { and } \quad p_{M}:=\sup _{Q_{2 r}} p(\cdot)
$$

and $d\left(p_{m}\right)$ is defined according to $(2.9)$ with $p_{m}$ instead of $p\left(\mathfrak{z}_{0}\right)$. For fixed $r \leq r_{1}<r_{2} \leq 2 r$ we consider the concentric parabolic cylinders

$$
Q_{r} \subseteq Q_{r_{1}} \subset Q_{r_{2}} \subseteq Q_{2 r}
$$

Then, for $\lambda \geq \lambda_{0}$ and $z_{0} \in Q_{r_{1}}$, parabolic cylinders of the type $Q_{s}^{(\lambda)}\left(z_{0}\right)$ with $s \leq \min \left\{\lambda^{\frac{p_{m}-2}{2 p_{m}}}, 1\right\}\left(r_{2}-r_{1}\right) / 2$ are contained in $Q_{r_{2}}$. With the help of a stopping time argument we now want to construct an appropriate cylinder around $z_{0}$ on which the conditions (6.1) and (6.2) are fulfilled and we therefore may apply Lemma 6.1. At first, we shall be concerned with the validity of (6.1). By Lebesgue's differentiation theorem for a.e. $z_{0} \in Q_{r_{1}}$ satisfying $\left|D u\left(z_{0}\right)\right|^{p_{0}}>\lambda$ we have:

$$
\lim _{s \downarrow 0} \int_{Q_{s}^{(\lambda)}\left(z_{0}\right)}(|D u|+|F|+1)^{p(\cdot)} d z \geq\left|D u\left(z_{0}\right)\right|^{p_{0}}>\lambda .
$$


This can be seen as follows: We know that in the Lebesgue points $z_{0}$ of $D u$ we have (cf. [20], [25], [26])

$$
\lim _{s \downarrow 0} \int_{Q_{s}\left(z_{0}\right)}|D u|^{p(\cdot)} d z=\left|D u\left(z_{0}\right)\right|^{p_{0}} .
$$

Note here, that the means are taken with respect to the usual parabolic cylinders while in (7.9) we need to have means on cylinders $Q_{s}^{(\lambda)}\left(z_{0}\right)$. Since $Q_{s}^{(\lambda)}\left(z_{0}\right) \subseteq Q_{\mu s}\left(z_{0}\right)$ for $\mu \equiv \max \left\{\lambda^{\frac{2-p_{0}}{2 p_{0}}}, 1\right\}$ we have

$$
\begin{aligned}
\left.\lim _{s \downarrow 0} f_{Q_{s}^{(\lambda)}\left(z_{0}\right)}|| D u\right|^{p(\cdot)}-\left|D u\left(z_{0}\right)\right|^{p_{0}} \mid d z & \\
& \leq\left.\frac{\mu^{n+2}}{\lambda^{\frac{2-p_{0}}{p_{0}}}} \lim _{s \downarrow 0} f_{Q_{\mu s}\left(z_{0}\right)}|| D u\right|^{p(\cdot)}-\left|D u\left(z_{0}\right)\right|^{p_{0}} \mid d z=0 .
\end{aligned}
$$

In the following we denote by $\chi \equiv \chi\left(n, L_{1}, \gamma_{1}\right) \geq 5$ the corresponding constant from Lemma 7.1. We let

$$
B^{\frac{1}{d\left(p_{m}\right)}+\frac{2}{p_{M}}-\frac{2}{p_{m}}}:=\left(\frac{8 \chi r}{r_{2}-r_{1}}\right)^{n+2}
$$

and consider values of $\lambda$ satisfying

$$
\lambda>B \lambda_{0}
$$

and radii $s$ with

$$
\frac{\min \left\{\lambda^{\frac{p_{m}-2}{2 p_{m}}}, 1\right\}\left(r_{2}-r_{1}\right)}{4 \chi} \leq s \leq \frac{\min \left\{\lambda^{\frac{p_{m}-2}{2 p_{m}}}, 1\right\}\left(r_{2}-r_{1}\right)}{2}
$$

Recalling the definition of $\lambda_{0}$ we get by enlarging the domain of integration from $Q_{s}^{(\lambda)}\left(z_{0}\right)$ to $Q_{2 r}$ the following estimate:

$$
\begin{aligned}
f_{Q_{s}^{(\lambda)}\left(z_{0}\right)}(|D u|+|F|+1)^{p(\cdot)} d z & \leq \frac{\left|Q_{2 r}\right|}{\left|Q_{s}^{(\lambda)}\left(z_{0}\right)\right|} f_{Q_{2 r}}(|D u|+|F|+1)^{p(\cdot)} d z \\
& =\left(\frac{2 r}{s}\right)^{n+2} \lambda^{\frac{p_{0}-2}{p_{0}}} \lambda_{0}^{\frac{1}{d\left(p_{m}\right)}+\frac{2}{p_{M}}-\frac{2}{p_{m}}} .
\end{aligned}
$$

In order to estimate the right-hand side from above we shall distinguish the cases $2 \leq p_{m} \leq \gamma_{2}$ and $\gamma_{1} \leq p_{m}<2$. When $p_{m} \geq 2$ we have $d\left(p_{m}\right)=\frac{p_{m}}{2}$ and $\min \left\{\lambda^{\frac{p_{m}-2}{2 p_{m}}}, 1\right\}=1$ and therefore by the definition of $B$ 
from (7.10) and the choices of $\lambda$ and $s$ as in (7.11) and (7.12) we get

$$
\begin{aligned}
f_{Q_{s}^{(\lambda)}\left(z_{0}\right)}(|D u|+|F|+1)^{p(\cdot)} d z & \leq\left(\frac{8 \chi r}{r_{2}-r_{1}}\right)^{n+2} \lambda^{\frac{p_{0}-2}{p_{0}}} \lambda_{0}^{\frac{2}{p_{M}}} \\
& <\left(\frac{8 \chi r}{r_{2}-r_{1}}\right)^{n+2} \frac{\lambda}{B^{\frac{2}{p_{M}}}}=\lambda .
\end{aligned}
$$

When $\gamma_{1} \leq p_{m}<2$ we have $\frac{1}{d\left(p_{m}\right)}=\frac{n+2}{2}-\frac{n}{p_{m}}$ and $\min \left\{\lambda^{\frac{p_{m}-2}{2 p_{m}}}, 1\right\}=$ $\lambda^{\frac{p_{m}-2}{2 p_{m}}}$. Arguing as for (7.13) we deduce that

$$
\begin{aligned}
& f_{Q_{s}^{(\lambda)}\left(z_{0}\right)}(|D u|+|F|+1)^{p(\cdot)} d z \\
& \leq\left(\frac{8 \chi r}{\lambda^{\frac{p_{m}-2}{2 p_{m}}}\left(r_{2}-r_{1}\right)}\right)^{n+2} \lambda^{\frac{p_{0}-2}{p_{0}}} \lambda_{0}^{\frac{1}{d\left(p_{m}\right)}+\frac{2}{p_{M}}-\frac{2}{p_{m}}} \\
& =\left(\frac{8 \chi r}{r_{2}-r_{1}}\right)^{n+2} \lambda^{\frac{2}{p_{m}}-\frac{1}{d\left(p_{m}\right)}} \lambda^{\frac{p_{0}-2}{p_{0}}} \lambda_{0}^{\frac{1}{d\left(p_{m}\right)}+\frac{2}{p_{M}}-\frac{2}{p_{m}}} \\
& \leq\left(\frac{8 \chi r}{r_{2}-r_{1}}\right)^{n+2} \lambda^{1+\frac{2}{p_{m}}-\frac{2}{p_{M}}-\frac{1}{d\left(p_{m}\right)}} \lambda_{0}^{\frac{1}{d\left(p_{m}\right)}+\frac{2}{p_{M}}-\frac{2}{p_{m}}} \\
& <\left(\frac{8 \chi r}{r_{2}-r_{1}}\right)^{n+2} \frac{\lambda}{B^{\frac{1}{d\left(p_{m}\right)}+\frac{2}{p_{M}}-\frac{2}{p_{m}}}}=\lambda .
\end{aligned}
$$

Therefore, (7.13), respectively (7.14) and (7.9) yield on the one hand a cylinder, namely $Q_{s}^{(\lambda)}\left(z_{0}\right)$, on which the integral possesses a value larger than $\lambda^{p_{0}}$, and on the other hand a cylinder on which the value of the integral is smaller than $\lambda^{p_{0}}$. More precisely, according to (7.9) and (7.13) when $p_{m} \geq 2$, respectively (7.14) when $p_{m}<2$ the absolute continuity of the integral yields the existence of a maximal radius $0<$ $\varrho_{z_{0}}<\min \left\{\lambda^{\frac{p_{m}-2}{2 p_{m}}}, 1\right\}\left(r_{2}-r_{1}\right) /(4 \chi)$ such that

$$
f_{Q_{\varrho z_{0}}^{(\lambda)}\left(z_{0}\right)}(|D u|+|F|+1)^{p(\cdot)} d z=\lambda
$$

while for any $s \in\left(\varrho_{z_{0}}, \min \left\{\lambda^{\frac{p_{m}-2}{2 p_{m}}}, 1\right\}\left(r_{2}-r_{1}\right) / 2\right]$ there holds

$$
f_{Q_{s}^{(\lambda)}\left(z_{0}\right)}(|D u|+|F|+1)^{p(\cdot)} d z<\lambda .
$$


At this stage we note that $Q_{2 \chi \varrho_{z_{0}}}^{(\lambda)}\left(z_{0}\right) \subseteq Q_{r_{2}}$ and therefore by (7.15) and (7.16) for $s=8 \varrho_{z_{0}}$ we conclude, that the assumptions (6.1) and (6.2) of Lemma 6.1 are fulfilled. Note here that $8 \leq 2 \chi$ and therefore $8 \varrho_{z_{0}} \in$ $\left(\varrho_{z_{0}}, \min \left\{\lambda^{\frac{p_{m}-2}{2 p_{m}}}, 1\right\}\left(r_{2}-r_{1}\right) / 2\right]$. In order to proceed further and to apply Lemma 6.1 we need to impose a bound on the radius $r$ of the form

$$
r \leq r_{0} \equiv r_{0}\left(n, L_{1}, \gamma_{1}, \gamma_{2}, M\right)
$$

Here $M$ is the upper bound for the total energy from (2.7) and $r_{0}$ denotes the radius bound from Lemma 6.1 (i.e. $r_{0} \equiv \varrho_{0}$ where $\varrho_{0}$ is from Lemma 6.1). Then, we are allowed to apply Lemma 6.1 yielding the following Reverse-Hölder inequality:

$$
\begin{aligned}
f_{Q_{\varrho z_{0}}^{(\lambda)}\left(z_{0}\right)}|D u|^{p(\cdot)} d z \leq c\left(f_{Q_{2 \varrho z_{0}}^{(\lambda)}\left(z_{0}\right)}|D u|^{\frac{p(\cdot)}{\vartheta}} d z\right)^{\vartheta} \\
\\
+c f_{Q_{2 \varrho_{0}}^{(\lambda)}\left(z_{0}\right)}(|F|+1)^{p(\cdot)} d z,
\end{aligned}
$$

where $\vartheta$ has been defined in (6.4) and $c \equiv c\left(n, N, \nu, L, L_{1}, \gamma_{1}, \gamma_{2}\right)$.

Now, for $\eta \in(0,1)$ to be fixed later we consider the upper level sets of $|D u|$ and $|F|+1$ defined by

$$
\Phi_{\eta \lambda}^{r_{1}}:=\left\{z \in Q_{r_{1}}:|D u(z)|^{p(z)}>\eta \lambda\right\}
$$

and

$$
\Psi_{\eta \lambda}^{r_{1}}:=\left\{z \in Q_{r_{1}}:(|F(z)|+1)^{p(z)}>\eta \lambda\right\}
$$

If $\eta \lambda>B \lambda_{0}$, then for a.e. $z_{0} \in \Phi_{\eta \lambda}^{r_{1}}$ there exists a parabolic cylin$\operatorname{der} Q_{\varrho_{z_{0}}}^{(\lambda)}\left(z_{0}\right)$ on which (7.15), (7.16) and (7.17) hold, and, moreover that $Q_{2 \chi \varrho_{z_{0}}}^{(\lambda)}\left(z_{0}\right) \subseteq Q_{r_{2}}$. We let

$$
p_{0} \equiv p\left(z_{0}\right), \quad p_{1} \equiv \inf _{Q_{2 \varrho z_{0}}^{(\lambda)}\left(z_{0}\right)} p(\cdot) \quad \text { and } \quad p_{2} \equiv \sup _{Q_{2 \varrho z_{0}}^{(\lambda)}\left(z_{0}\right)} p(\cdot) .
$$

Our next aim is to infer a suitable estimate for the $L^{p(\cdot)}$-norm of $D u$ on the cylinder $Q_{2 \chi \varrho_{z_{0}}}^{(\lambda)}\left(z_{0}\right)$. Using in turn $(7.17),(7.15)$ and Hölder's 
238

V. Bögelein, F. DuzaAr

inequality we obtain:

$$
\begin{aligned}
& f_{Q_{\varrho z_{0}}^{(\lambda)}\left(z_{0}\right)}(|D u|+|F|+1)^{p(\cdot)} d z \\
& \leq 2^{\gamma_{2}-1} f_{Q_{\varrho z_{0}}^{(\lambda)}\left(z_{0}\right)}|D u|^{p(\cdot)} d z+2^{\gamma_{2}-1} f_{Q_{\varrho z_{0}}^{(\lambda)}\left(z_{0}\right)}(|F|+1)^{p(\cdot)} d z \\
& \leq c\left(f_{Q_{2 \varrho_{z_{0}}}^{(\lambda)}\left(z_{0}\right)}|D u|^{\frac{p(\cdot)}{\vartheta}} d z\right)^{\vartheta}+c f_{Q_{2 \varrho_{z_{0}}}^{(\lambda)}\left(z_{0}\right)}(|F|+1)^{p(\cdot)} d z \\
& \leq c 2^{\vartheta-1} \eta \lambda+c 2^{\vartheta-1}\left(\frac{1}{\left|Q_{2 \varrho_{z_{0}}}^{(\lambda)}\left(z_{0}\right)\right|} \int_{Q_{2 \varrho_{z_{0}}}^{(\lambda)}\left(z_{0}\right) \cap \Phi_{\eta \lambda}^{r_{2}}}|D u|^{\frac{p(\cdot)}{\vartheta}} d z\right)^{\vartheta} \\
& +c \eta \lambda+\frac{c}{\left|Q_{2 \varrho_{z_{0}}}^{(\lambda)}\left(z_{0}\right)\right|} \int_{Q_{2 \varrho_{z_{0}}}^{(\lambda)}\left(z_{0}\right) \cap \Psi_{\eta \lambda}^{r_{2}}}(|F|+1)^{p(\cdot)} d z \\
& \leq c \eta \lambda \\
& +\frac{c}{\left|Q_{2 \varrho_{z_{0}}}^{(\lambda)}\left(z_{0}\right)\right|} \int_{Q_{2 \varrho z_{0}}^{(\lambda)}\left(z_{0}\right) \cap \Phi_{\eta \lambda}^{r 2}}|D u|^{\frac{p(\cdot)}{\vartheta}} d z\left(f_{Q_{2 \varrho z_{0}}^{(\lambda)}\left(z_{0}\right)}|D u|^{\frac{p(\cdot)}{\vartheta}} d z\right)^{\vartheta-1} \\
& +\frac{c}{\left|Q_{2 \varrho_{z_{0}}}^{(\lambda)}\left(z_{0}\right)\right|} \int_{Q_{2 \varrho z_{0}}^{(\lambda)}\left(z_{0}\right) \cap \Psi_{\eta \lambda}^{r_{2}}}(|F|+1)^{p(\cdot)} d z \\
& =c \eta f_{Q_{\varrho z_{0}}^{(\lambda)}\left(z_{0}\right)}(|D u|+|F|+1)^{p(\cdot)} d z
\end{aligned}
$$

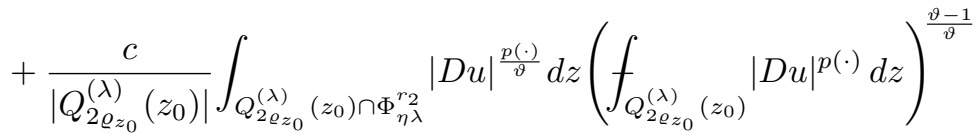

$$
\begin{aligned}
& +\frac{c}{\left|Q_{2 \varrho_{z_{0}}}^{(\lambda)}\left(z_{0}\right)\right|} \int_{Q_{2 \varrho z_{0}}^{(\lambda)}\left(z_{0}\right) \cap \Psi_{\eta \lambda}^{r_{2}}}(|F|+1)^{p(\cdot)} d z
\end{aligned}
$$

where $c=c\left(n, \nu, L, L_{1}, \gamma_{1}, \gamma_{2}\right)$. Choosing $\eta \equiv \eta\left(n, \nu, L, L_{1}, \gamma_{1}, \gamma_{2}\right)>$ 0 small enough - ie. of the form $\eta \equiv 1 /(2 c)$ - we can reabsorb the first integral appearing on the right-hand side into the left. Moreover, 
from (7.16) we know that

$$
f_{Q_{2 \varrho z_{0}}^{(\lambda)}\left(z_{0}\right)}|D u|^{p(\cdot)} d z<\lambda .
$$

Proceeding in this way and multiplying the resulting inequality by $\left|Q_{2 \varrho_{z_{0}}}^{(\lambda)}\left(z_{0}\right)\right|$ we obtain

$$
\begin{aligned}
\int_{Q_{\varrho z_{0}}^{(\lambda)}\left(z_{0}\right)}(|D u|+|F|+1)^{p(\cdot)} d z \leq & c \int_{Q_{2 \varrho z_{0}}^{(\lambda)}\left(z_{0}\right) \cap \Phi_{\eta \lambda}^{r_{2}}} \lambda^{\frac{\vartheta-1}{\vartheta}}|D u|^{\frac{p(\cdot)}{\vartheta}} d z \\
& +c \int_{Q_{2 \varrho z_{0}}^{(\lambda)}\left(z_{0}\right) \cap \Psi_{\eta \lambda}^{r_{2}}}(|F|+1)^{p(\cdot)} d z
\end{aligned}
$$

where $c \equiv c\left(n, \nu, L, L_{1}, \gamma_{1}, \gamma_{2}\right)$. Using in turn (7.15) and (7.16) with $s=$ $2 \chi \varrho_{z_{0}}$ we can bound the left-hand side of the preceding inequality from below by

which leads us to

$$
f_{Q_{2 \chi \varrho z_{0}}^{(\lambda)}\left(z_{0}\right)}|D u|^{p(\cdot)} d z
$$

$$
\begin{aligned}
\int_{Q_{2 \chi \varrho z_{0}}^{(\lambda)}\left(z_{0}\right)}|D u|^{p(\cdot)} d z \leq c \int_{Q_{2 \varrho z_{0}}^{(\lambda)}\left(z_{0}\right) \cap \Phi_{\eta \lambda}^{r_{2}}} \lambda^{\frac{\vartheta-1}{\vartheta}}|D u|^{\frac{p(\cdot)}{\vartheta}} d z \\
+c \int_{Q_{2 \varrho z_{0}}^{(\lambda)}\left(z_{0}\right) \cap \Psi_{\eta \lambda}^{r_{2}}}(|F|+1)^{p(\cdot)} d z
\end{aligned}
$$

for a constant $c \equiv c\left(n, N, \nu, L, L_{1}, \gamma_{1}, \gamma_{2}\right)$.

At this stage we impose a further bound for the radii $r \leq r_{0}$ of the following form:

$$
r \leq r_{0} \leq\left[6 \sqrt{\beta_{n} M}\right]^{-\frac{2}{\alpha}},
$$

where $\alpha$ is defined in (3.3). Moreover, we note that (7.15) implies (3.1) with $\kappa=1$ and therefore from (3.2) we get

$$
\lambda \leq\left(\frac{\beta_{n} M}{\varrho_{z_{0}}^{n+2}}\right)^{\frac{p_{0}}{2}},
$$

where $\beta_{n} \equiv \beta_{n}(n)$.

Thus, so far we have shown that for any $\lambda>B \lambda_{0}$ the level set $\Phi_{\lambda}^{r_{1}}$ is covered by a family $\mathcal{F} \equiv\left\{Q_{2 \chi \varrho_{z_{0}}}^{(\lambda)}\left(z_{0}\right)\right\}$ of parabolic cylinders with center $z_{0} \in \Phi_{\lambda}^{r_{1}}$ whose radii $\varrho_{z_{0}}$ are bounded by $\left[\left(\beta_{n} M\right)^{\frac{p_{0}}{2}} \lambda^{-1}\right]^{\frac{2}{p_{0}(n+2)}}$ which is a consequence of (7.20) and the radius $r_{0}$ from (7.19). Furthermore, 
on each cylinder of the covering we have (7.18) at our hands. From Vitali's covering theorem, i.e. the version for non-uniformly parabolic cylinders, provided in Lemma 7.1 we infer the existence of a countable subfamily $\left\{Q_{2 \varrho_{z_{i}}}^{(\lambda)}\left(z_{i}\right)\right\}_{i=1}^{\infty} \subseteq \mathcal{F}$ of pair-wise disjoint parabolic cylinders, such that the $\chi$-times enlarged cylinders $Q_{2 \chi \varrho_{z_{i}}}^{(\lambda)}\left(z_{i}\right)$ cover the set $\Phi_{\lambda}^{r_{1}}$, i.e. up to a set of measure zero there holds (note that $Q_{2 \chi \varrho_{z_{i}}}^{(\lambda)}\left(z_{i}\right) \subseteq Q_{r_{2}}$ by construction)

$$
\Phi_{\lambda}^{r_{1}} \subseteq \bigcup_{i=1}^{\infty} Q_{2 \chi \varrho_{z_{i}}}^{(\lambda)}\left(z_{i}\right) \subseteq Q_{r_{2}}
$$

Recalling that the cylinders $\left\{Q_{2 \varrho_{z_{i}}}^{(\lambda)}\left(z_{i}\right)\right\}_{i=1}^{\infty}$ are pair-wise disjoint we infer from (7.18) that

$$
\begin{aligned}
& \int_{\Phi_{\lambda}^{r_{1}}}|D u|^{p(\cdot)} d z \leq \sum_{i=1}^{\infty} \int_{Q_{2 \chi \varrho z_{i}}^{(\lambda)}\left(z_{i}\right)}|D u|^{p(\cdot)} d z \\
& \leq c \sum_{i=1}^{\infty}\left[\int_{Q_{2 \varrho z_{i}}^{(\lambda)}\left(z_{i}\right) \cap \Phi_{\eta \lambda}^{r_{2}}} \lambda^{\frac{\vartheta-1}{\vartheta}}|D u|^{\frac{p(\cdot)}{\vartheta}} d z\right. \\
& \left.+\int_{Q_{2 \varrho_{z_{i}}}^{(\lambda)}\left(z_{i}\right) \cap \Psi_{\eta \lambda}^{r_{2}}}(|F|+1)^{p(\cdot)} d z\right] \\
& \leq c \int_{\Phi_{\eta \lambda}^{r_{2}}} \lambda^{\frac{\vartheta-1}{\vartheta}}|D u|^{\frac{p(\cdot)}{\vartheta}} d z+c \int_{\Psi_{\eta \lambda}^{r_{2}}}(|F|+1)^{p(\cdot)} d z,
\end{aligned}
$$

with a constant $c \equiv c\left(n, N, \nu, L, L_{1}, \gamma_{1}, \gamma_{2}\right)$. Moreover, on $\Phi_{\eta \lambda}^{r_{1}} \backslash \Phi_{\lambda}^{r_{1}}$ we have $|D u|^{p(\cdot)} \leq \lambda$ and therefore

$$
\int_{\Phi_{\eta \lambda}^{r_{1}} \backslash \Phi_{\lambda}^{r_{1}}}|D u|^{p(\cdot)} d z \leq \int_{\Phi_{\eta \lambda}^{r_{1}} \backslash \Phi_{\lambda}^{r_{1}}} \lambda^{\frac{\vartheta-1}{\vartheta}}|D u|^{\frac{p(\cdot)}{\vartheta}} d z \leq \int_{\Phi_{\eta \lambda}^{r_{1}}} \lambda^{\frac{\vartheta-1}{\vartheta}}|D u|^{\frac{p(\cdot)}{\vartheta}} d z .
$$

Combining this with the second last estimate we infer the following Reverse-Hölder inequality for super-level sets with increasing domains:

$$
\int_{\Phi_{\eta \lambda}^{r_{1}}}|D u|^{p(\cdot)} d z \leq c \int_{\Phi_{\eta \lambda}^{r_{2}}} \lambda^{\frac{\vartheta-1}{\vartheta}}|D u|^{\frac{p(\cdot)}{\vartheta}} d z+c \int_{\Psi_{\eta \lambda}^{r_{2}}}(|F|+1)^{p(\cdot)} d z,
$$

where $c \equiv c\left(n, N, \nu, L, L_{1}, \gamma_{1}, \gamma_{2}\right)$. Here we replace $\eta \lambda$ by $\lambda$ and recall that $\eta<1$ depends only on $n, \nu, L, L_{1}, \gamma_{1}, \gamma_{2}$. We obtain for any $\lambda \geq$ 
$B \lambda_{0} / \eta \equiv \lambda_{1}$ that

$$
\begin{aligned}
\int_{\Phi_{\lambda}^{r_{1}}}|D u|^{p(\cdot)} d z & \leq c \eta^{-\frac{\vartheta-1}{\vartheta}} \int_{\Phi_{\lambda}^{r_{2}}} \lambda^{\frac{\vartheta-1}{\vartheta}}|D u|^{\frac{p(\cdot)}{\vartheta}} d z+c \int_{\Psi_{\lambda}^{r_{2}}}(|F|+1)^{p(\cdot)} d z \\
& \leq c \int_{\Phi_{\lambda}^{r_{2}}} \lambda^{\frac{\vartheta-1}{\vartheta}}|D u|^{\frac{p(\cdot)}{\vartheta}} d z+c \int_{\Psi_{\lambda}^{r_{2}}}(|F|+1)^{p(\cdot)} d z
\end{aligned}
$$

for a constant $c=c\left(n, N, \nu, L, L_{1}, \gamma_{1}, \gamma_{2}\right)$. Having arrived at this stage we would like to multiply the preceding inequality by $\lambda^{\varepsilon-1}$ with $\varepsilon \in(0,1]$ and then integrate with respect to $\lambda$ over $\left(\lambda_{1}, \infty\right)$. This, formally, would lead in a standard way to the desired higher-integrability of $|D u|$, where $\varepsilon$ has to be chosen small enough in between in order to re-absorb certain terms on the left-hand side. However, there is a difficulty in moving terms to the left-hand side since they may be infinite. This technical problem can be treated, for example, by truncating $|D u|$ (see [29] for example). The precise argument is as follows: For $k>\lambda_{1}$ we define

$|D u|_{k}:=\min \{|D u|, k\} \quad$ and $\quad \Phi_{\lambda, k}^{r_{i}}:=\left\{z \in Q_{r_{i}}:|D u(z)|_{k}^{p(z)}>\lambda\right\}, \quad i=1,2$

and deduce from the preceding inequality that

$$
\begin{aligned}
\int_{\Phi_{\lambda, k}^{r_{1}}}|D u|_{k}^{\frac{p(\cdot)(\vartheta-1)}{\vartheta}}|D u|^{\frac{p(\cdot)}{\vartheta}} d z \leq c \int_{\Phi_{\lambda, k}^{r_{2}}} \lambda^{\frac{\vartheta-1}{\vartheta}}|D u|^{\frac{p(\cdot)}{\vartheta}} d z \\
+c \int_{\Psi_{\lambda}^{r_{2}}}(|F|+1)^{p(\cdot)} d z .
\end{aligned}
$$

Note that (7.21) trivially holds when $k \leq \lambda$ since in this case $\Phi_{\lambda, k}^{r_{1}}=\emptyset$, while in the case $k>\lambda$ (7.21) follows from the second last inequality since $\Phi_{\lambda, k}^{r_{2}} \equiv \Phi_{\lambda}^{r_{2}}$ and $|D u|_{k} \leq|D u|$. Now, we multiply (7.21) by $\lambda^{\varepsilon-1}$, where $\varepsilon \in(0,1]$ will be fixed later, and integrate with respect to $\lambda$ over $\left(\lambda_{1}, \infty\right)$. We obtain

$$
\begin{aligned}
\int_{\lambda_{1}}^{\infty} \lambda^{\varepsilon-1} \int_{\Phi_{\lambda, k}^{r_{1}}}|D u|_{k}^{\frac{p(\cdot)(\vartheta-1)}{\vartheta}}|D u|^{\frac{p(\cdot)}{\vartheta}} d z d \lambda \\
\leq c \int_{\lambda_{1}}^{\infty} \int_{\Phi_{\lambda, k}^{r_{2}}} \lambda^{\frac{\vartheta-1}{\vartheta}+\varepsilon-1}|D u|^{\frac{p(\cdot)}{\vartheta}} d z d \lambda \\
+c \int_{\lambda_{1}}^{\infty} \lambda^{\varepsilon-1} \int_{\Psi_{\lambda}^{r_{2}}}(|F|+1)^{p(\cdot)} d z d \lambda .
\end{aligned}
$$


To the integral on the left-hand side we apply Fubini's theorem and find that

$$
\begin{aligned}
\int_{\lambda_{1}}^{\infty} & \lambda^{\varepsilon-1} \int_{\Phi_{\lambda, k}^{r_{1}}}|D u|_{k}^{\frac{p(\cdot)(\vartheta-1)}{\vartheta}}|D u|^{\frac{p(\cdot)}{\vartheta}} d z d \lambda \\
\quad & \int_{\Phi_{\lambda_{1}, k}^{r_{1}}}|D u|_{k}^{\frac{p(\cdot)(\vartheta-1)}{\vartheta}}|D u|^{\frac{p(\cdot)}{\vartheta}} \int_{\lambda_{1}}^{|D u(z)|_{k}^{p(z)}} \lambda^{\varepsilon-1} d \lambda d z \\
& =\frac{1}{\varepsilon} \int_{\Phi_{\lambda_{1}, k}^{r_{1}}}\left[|D u|_{k}^{\frac{p(\cdot)(\vartheta-1)}{\vartheta}+\varepsilon p(\cdot)}|D u|^{\frac{p(\cdot)}{\vartheta}}-\lambda_{1}^{\varepsilon}|D u|_{k}^{\frac{p(\cdot)(\vartheta-1)}{\vartheta}}|D u|^{\frac{p(\cdot)}{\vartheta}}\right] d z
\end{aligned}
$$

The first integral on the right-hand side is treated similarly. Here we obtain that

$$
\begin{aligned}
\int_{\lambda_{1}}^{\infty} \int_{\Phi_{\lambda, k}^{r_{2}}} \lambda^{\frac{\vartheta-1}{\vartheta}+\varepsilon-1}|D u|^{\frac{p(\cdot)}{\vartheta}} d z d \lambda \\
\quad=\int_{\Phi_{\lambda_{1}, k}^{r_{2}}}|D u|^{\frac{p(\cdot)}{\vartheta}} \int_{\lambda_{1}}^{|D u(z)|_{k}^{p(z)}} \lambda^{\frac{\vartheta-1}{\vartheta}+\varepsilon-1} d \lambda d z \\
\leq \int_{\Phi_{\lambda_{1}, k}^{r_{2}}} \frac{1}{\frac{\vartheta-1}{\vartheta}+\varepsilon}|D u|_{k}^{\frac{p(\cdot)(\vartheta-1)}{\vartheta}+\varepsilon p(\cdot)}|D u|^{\frac{p(\cdot)}{\vartheta}} d z \\
\leq \frac{\vartheta}{\vartheta-1} \int_{\Phi_{\lambda_{1}, k}^{r_{2}}}|D u|_{k}^{\frac{p(\cdot)(\vartheta-1)}{\vartheta}+\varepsilon p(\cdot)}|D u|^{\frac{p(\cdot)}{\vartheta}} d z .
\end{aligned}
$$

Finally, for the second integral on the right-hand side we get

$$
\begin{aligned}
\int_{\lambda_{1}}^{\infty} \lambda^{\varepsilon-1} \int_{\Psi_{\lambda}^{r_{2}}}(|F|+1)^{p(\cdot)} d z d \lambda \\
\quad=\int_{\Psi_{\lambda_{1}}^{r_{2}}}(|F|+1)^{p(\cdot)} \int_{\lambda_{1}}^{(|F(z)|+1)^{p(z)}} \lambda^{\varepsilon-1} d \lambda d z \\
\leq \frac{1}{\varepsilon} \int_{\Psi_{\lambda_{2}}^{r_{2}}}(|F|+1)^{p(\cdot)(1+\varepsilon)} d z
\end{aligned}
$$


Joining the preceding estimates with (7.22) and multiplying by $\varepsilon$ we arrive at

$$
\begin{aligned}
\int_{\Phi_{\lambda_{1}, k}^{r_{1}}} & |D u|_{k}^{\frac{p(\cdot)(\vartheta-1)}{\vartheta}+\varepsilon p(\cdot)}|D u|^{\frac{p(\cdot)}{\vartheta}} d z \\
\leq & \lambda_{1}^{\varepsilon} \int_{\Phi_{\lambda_{1}, k}^{r_{1}}}|D u|_{k}^{\frac{p(\cdot)(\vartheta-1)}{\vartheta}}|D u|^{\frac{p(\cdot)}{\vartheta}} d z+c \int_{\Psi_{\lambda_{1}}^{r_{2}}}(|F|+1)^{p(\cdot)(1+\varepsilon)} d z \\
& +\frac{\varepsilon c \vartheta}{\vartheta-1} \int_{\Phi_{\lambda_{1}, k}^{r_{2}}}|D u|_{k}^{\frac{p(\cdot)(\vartheta-1)}{\vartheta}+\varepsilon p(\cdot)}|D u|^{\frac{p(\cdot)}{\vartheta}} d z
\end{aligned}
$$

for a constant $c=c\left(n, N, \nu, L, L_{1}, \gamma_{1}, \gamma_{2}\right)$. Using

$$
\begin{aligned}
& \int_{Q_{r_{1}} \backslash \Phi_{\lambda_{1}, k}^{r_{1}}}|D u|_{k}^{\frac{p(\cdot)(\vartheta-1)}{\vartheta}+\varepsilon p(\cdot)}|D u|^{\frac{p(\cdot)}{\vartheta}} d z \\
& \leq \lambda_{1}^{\varepsilon} \int_{Q_{r_{1}} \backslash \Phi_{\lambda_{1}, k}^{r_{1}}}|D u|_{k}^{\frac{p(\cdot)(\vartheta-1)}{\vartheta}}|D u|^{\frac{p(\cdot)}{\vartheta}} d z
\end{aligned}
$$

we obtain

$$
\begin{aligned}
\int_{Q_{r_{1}}}|D u|_{k}^{\frac{p(\cdot)(\vartheta-1)}{\vartheta}+\varepsilon p(\cdot)}|D u|^{\frac{p(\cdot)}{\vartheta}} d z \leq & \frac{\varepsilon c \vartheta}{\vartheta-1} \int_{Q_{r_{2}}}|D u|_{k}^{\frac{p(\cdot)(\vartheta-1)}{\vartheta}+\varepsilon p(\cdot)}|D u|^{\frac{p(\cdot)}{\vartheta}} d z \\
& +\lambda_{1}^{\varepsilon} \int_{Q_{2 r}}|D u|_{k}^{\frac{p(\cdot)(\vartheta-1)}{\vartheta}}|D u|^{\frac{p(\cdot)}{\vartheta}} d z \\
& +c \int_{Q_{2 r}}(|F|+1)^{p(\cdot)(1+\varepsilon)} d z .
\end{aligned}
$$

At this stage we perform the choice of $\varepsilon$. Choosing

$$
0<\varepsilon \leq \varepsilon_{0} \equiv \varepsilon_{0}\left(n, N, \nu, L, L_{1}, \gamma_{1}, \gamma_{2}, \sigma\right) \equiv \min \{\sigma,(\vartheta-1) /(2 c \vartheta)\},
$$

recalling the definitions of $\lambda_{1}$, i.e. $\lambda_{1}^{\varepsilon}=\left(B \lambda_{0} / \eta\right)^{\varepsilon} \leq B \lambda_{0}^{\varepsilon} / \eta$ since $B / \eta \geq$ $1, \varepsilon \leq 1$, and of $B$ from (7.10) we arrive at

$$
\begin{aligned}
\int_{Q_{r_{1}}}|D u|_{k}^{\frac{p(\cdot)(\vartheta-1)}{\vartheta}+\varepsilon p(\cdot)}|D u|^{\frac{p(\cdot)}{\vartheta}} d z \leq & \frac{1}{2} \int_{Q_{r_{2}}}|D u|_{k}^{\frac{p(\cdot)(\vartheta-1)}{\vartheta}+\varepsilon p(\cdot)}|D u|^{\frac{p(\cdot)}{\vartheta}} d z \\
& +\frac{c_{*}(2 r)^{\beta} \lambda_{0}^{\varepsilon}}{\left(r_{2}-r_{1}\right)^{\beta}} \int_{Q_{2 r}}|D u|_{k}^{\frac{p(\cdot)(\vartheta-1)}{\vartheta}}|D u|^{\frac{p(\cdot)}{\vartheta}} d z \\
& +c \int_{Q_{2 r}}(|F|+1)^{p(\cdot)(1+\varepsilon)} d z
\end{aligned}
$$


where

$$
c_{*} \equiv \frac{(4 \chi)^{\beta}}{\eta} \quad \text { and } \quad \beta \equiv \frac{n+2}{\frac{1}{d\left(p_{m}\right)}+\frac{2}{p_{M}}-\frac{2}{p_{m}}} .
$$

Since $r \leq r_{1}<r_{2} \leq 2 r$ are arbitrary we are in the position to apply Lemma 3.1 with $\beta, \bar{\vartheta}=\frac{1}{2}$,

$$
\phi(s) \equiv \int_{Q_{s}}|D u|_{k}^{\frac{p(\cdot)(\vartheta-1)}{\vartheta}+\varepsilon p(\cdot)}|D u|^{\frac{p(\cdot)}{\vartheta}} d z
$$

and

$$
\begin{aligned}
& A \equiv c_{*}(2 r)^{\beta} \lambda_{0}^{\varepsilon} \int_{Q_{2 r}}|D u|_{k}^{\frac{p(\cdot)(\vartheta-1)}{\vartheta}}|D u|^{\frac{p(\cdot)}{\vartheta}} d z, \\
& C \equiv c \int_{Q_{2 r}}(|F|+1)^{p(\cdot)(1+\varepsilon)} d z .
\end{aligned}
$$

This allows to move the first integral from the right-hand side to the left and leads to

$$
\begin{aligned}
& \int_{Q_{r}}|D u|_{k}^{\frac{p(\cdot)(\vartheta-1)}{\vartheta}+\varepsilon p(\cdot)}|D u|^{\frac{p(\cdot)}{\vartheta}} d z \\
& \leq c(\beta)\left[2^{\beta} c_{*} \lambda_{0}^{\varepsilon} \int_{Q_{2 r}}|D u|_{k}^{\frac{p(\cdot)(\vartheta-1)}{\vartheta}}|D u|^{\frac{p(\cdot)}{\vartheta}} d z+c \int_{Q_{2 r}}(|F|+1)^{p(\cdot)(1+\varepsilon)} d z\right] .
\end{aligned}
$$

Letting $k \rightarrow \infty$ (here we apply Fatou's lemma on the left-hand side and Lebesgue's dominated convergence theorem on the right-hand side integrals) we get

$$
\int_{Q_{r}}|D u|^{p(\cdot)(1+\varepsilon)} d z \leq c\left[\lambda_{0}^{\varepsilon} \int_{Q_{2 r}}|D u|^{p(\cdot)} d z+\int_{Q_{2 r}}(|F|+1)^{p(\cdot)(1+\varepsilon)} d z\right],
$$

for a constant $c \equiv c\left(n, N, \nu, L, L_{1}, \gamma_{1}, \gamma_{2}\right)$. At this stage we mention that the dependence on $\beta$ can be eliminated since $p_{m} \in\left[\gamma_{1}, \gamma_{2}\right]$ and $p \mapsto d(p)$ is a continuous map. In this way we have $c(\beta)=c\left(n, \gamma_{1}, \gamma_{2}\right)$. Finally, passing to averages and recalling the definition of $\lambda_{0}$, i.e. (7.8), we deduce that

$$
\begin{aligned}
f_{Q_{r}}|D u|^{p(\cdot)(1+\varepsilon)} d z \leq & c\left(f_{Q_{2 r}}(|D u|+|F|+1)^{p(\cdot)} d z\right)^{1+\varepsilon\left[\frac{1}{d\left(p_{m}\right)}+\frac{2}{p_{M}}-\frac{2}{p_{m}}\right]^{-1}} \\
& +c f_{Q_{2 r}}(|F|+1)^{p(\cdot)(1+\varepsilon)} d z
\end{aligned}
$$

Finally, we want to replace in the preceding estimate $\left[\frac{1}{d\left(p_{m}\right)}+\frac{2}{p_{M}}-\frac{2}{p_{m}}\right]^{-1}$ by $d\left(p_{0}\right)$, where $p_{0} \equiv p\left(\mathfrak{z}_{0}\right)$ denotes the value of $p(\cdot)$ evaluated at the 
center $\mathfrak{z}_{0}$ of $Q_{2 r} \equiv Q_{2 r}\left(\mathfrak{z}_{0}\right)$. Using (2.2) we obtain

$$
\begin{aligned}
0 \leq\left[\frac{1}{d\left(p_{m}\right)}+\frac{2}{p_{M}}-\frac{2}{p_{m}}\right]^{-1}-d\left(p_{0}\right) & \leq\left[\frac{1}{d\left(p_{m}\right)}-\frac{2 \omega(4 r)}{\gamma_{1}^{2}}\right]^{-1}-d\left(p_{0}\right) \\
& =\frac{d\left(p_{m}\right)}{1-2 \gamma_{1}^{-2} d\left(p_{m}\right) \omega(4 r)}-d\left(p_{0}\right) \\
& =\frac{d\left(p_{m}\right)-d\left(p_{0}\right)+2 \gamma_{1}^{-2} d\left(p_{m}\right) d\left(p_{0}\right) \omega(4 r)}{1-2 \gamma_{1}^{-2} d\left(p_{m}\right) \omega(4 r)} \\
& \leq \frac{d\left(p_{m}\right)-d\left(p_{0}\right)+2 \gamma_{1}^{-2} \mathcal{D}^{2} \omega(4 r)}{1-2 \gamma_{1}^{-2} \mathcal{D} \omega(4 r)}
\end{aligned}
$$

where we denoted $\mathcal{D}:=\max \left\{d\left(\gamma_{1}\right), d\left(\gamma_{2}\right)\right\}$. At this point we impose a further restriction on $r_{0}$ of the form:

$$
\omega\left(4 r_{0}\right) \leq \min \left\{\frac{\gamma_{1}^{2}}{4 \mathcal{D}}, \frac{2}{n+2}\right\}
$$

leading us to the bound

$$
\left[\frac{1}{d\left(p_{m}\right)}+\frac{2}{p_{M}}-\frac{2}{p_{m}}\right]^{-1}-d\left(p_{0}\right) \leq 2\left[d\left(p_{m}\right)-d\left(p_{0}\right)+2 \gamma_{1}^{-2} \mathcal{D}^{2} \omega(4 r)\right]
$$

It remains to bound the difference $d\left(p_{m}\right)-d\left(p_{0}\right)$. In the case $2 \leq p_{m} \leq p_{0}$ we have

$$
d\left(p_{m}\right)-d\left(p_{0}\right)=\frac{p_{m}}{2}-\frac{p_{0}}{2} \leq 0 .
$$

In the case $p_{m} \leq p_{0} \leq 2$ we have

$$
\begin{aligned}
d\left(p_{m}\right)-d\left(p_{0}\right) & =\frac{2 p_{m}}{p_{m}(n+2)-2 n}-\frac{2 p_{0}}{p_{0}(n+2)-2 n} \\
& =\frac{4 n\left(p_{0}-p_{m}\right)}{\left(p_{m}(n+2)-2 n\right)\left(p_{0}(n+2)-2 n\right)} \\
& \leq \frac{4 n \omega(4 r)}{\left[\left(\gamma_{1}(n+2)-2 n\right]^{2}\right.} .
\end{aligned}
$$


In the remaining case $p_{m}<2 \leq p_{0}$ we use the fact $p_{m} \geq p_{0}-\omega(4 r) \geq$ $2-\omega(4 r)$ and (7.24) to infer

$$
\begin{aligned}
d\left(p_{m}\right)-d\left(p_{0}\right) & =\frac{2 p_{m}}{p_{m}(n+2)-2 n}-\frac{p_{0}}{2} \\
& \leq \frac{2 p_{m}}{(2-\omega(4 r))(n+2)-2 n}-\frac{p_{m}}{2} \\
& =\frac{2 p_{m}}{4-\omega(4 r)(n+2)}-\frac{p_{m}}{2} \\
& =\frac{p_{m}(n+2) \omega(4 r)}{8-2 \omega(4 r)(n+2)} \\
& \leq \frac{\gamma_{2}(n+2) \omega(4 r)}{4} .
\end{aligned}
$$

Hence, in any case we have $d\left(p_{m}\right)-d\left(p_{0}\right) \leq c\left(n, \gamma_{1}, \gamma_{2}\right) \omega(4 r)$. Inserting this into (7.25) we conclude that

$$
0 \leq\left[\frac{1}{d\left(p_{m}\right)}+\frac{2}{p_{M}}-\frac{2}{p_{m}}\right]^{-1}-d\left(p_{0}\right) \leq c\left(n, \gamma_{1}, \gamma_{2}\right) \omega(4 r)
$$

The preceding estimate together with $\varepsilon \leq 1$ and (2.7) implies

$$
\begin{aligned}
\left(f_{Q_{2 r}}(|D u|+|F|+1)^{p(\cdot)} d z\right)^{\left.\varepsilon\left[\frac{1}{d\left(p_{m}\right)}+\frac{2}{p_{M}}-\frac{2}{p_{m}}\right]^{-1}-d\left(p_{0}\right)\right]} \\
\quad \leq\left(f_{Q_{2 r}}(|D u|+|F|+1)^{p(\cdot)} d z\right)^{c\left(n, \gamma_{1}, \gamma_{2}\right) \omega(4 r)} \\
\leq\left(c(n)(2 r)^{-(n+2) \omega(4 r)} M^{\omega(4 r)}\right)^{c\left(n, \gamma_{1}, \gamma_{2}\right)} \\
\leq c\left(n, \gamma_{1}, \gamma_{2}\right)\left((4 r)^{-\omega(4 r)} M^{\omega(4 r)}\right)^{c\left(n, \gamma_{1}, \gamma_{2}\right)} .
\end{aligned}
$$

In the last step we used $M \geq 1$. In order to proceed further we use the logarithmic continuity condition (2.5) twice to infer for the two terms in the last bracket that

$$
(4 r)^{-\omega(4 r)} \leq c\left(L_{1}\right) \quad \text { and } \quad M^{\omega(4 r)} \leq c\left(L_{1}\right) .
$$

The second assertion is obtained as follows:

$$
M^{\omega(4 r)}=\exp [\omega(4 r) \log M] \leq \exp [\omega(1 / M) \log M] \leq e^{L_{1}},
$$


provided $r \leq r_{0} \leq \frac{1}{4 M}$ This restriction on the size of $r_{0}$ is already implied by the restriction from (7.19). Joining the preceding estimates we find

$\left(f_{Q_{2 r}}(|D u|+|F|+1)^{p(\cdot)} d z\right)^{\varepsilon\left[\left[\frac{1}{d\left(p_{m}\right)}+\frac{2}{p_{M}}-\frac{2}{p_{m}}\right]^{-1}-d\left(p_{0}\right)\right]} \leq c\left(n, L_{1}, \gamma_{1}, \gamma_{2}\right)$,

which together with (7.23) yields the desired estimate (2.8). This finally completes the proof of Theorem 2.2.

\section{Possible extensions}

Using the same techniques we can also treat other types of right-hand sides as for example inhomogeneities depending on $\boldsymbol{D u}$. Here, we consider parabolic systems of the type

$$
\partial_{t} u-\operatorname{div} a(z, D u)=b(z, D u) \quad \text { in } \Omega_{T},
$$

where the vector-field $a$ satisfies (2.1), while the right-hand side fulfills

$$
|b(z, w)| \leq L(|w|+1)^{p(z)-1},
$$

whenever $z \in \Omega_{T}$ and $w \in \mathbb{R}^{N n}$. In this setting, we can show again the higher integrability statement of Theorem 2.2. The proof can be achieved by simple modifications, since the terms resulting from the inhomogeneity and containing $D u$ can be re-absorbed into the left-hand side in the course of the proof of the Caccioppoli inequality; cf. [29].

\section{References}

[1] E. Acerbi And G. Mingione, Regularity results for a class of functionals with non-standard growth, Arch. Ration. Mech. Anal. 156(2) (2001), 121-140.

[2] E. Acerbi And G. Mingione, Regularity results for stationary electro-rheological fluids, Arch. Ration. Mech. Anal. 164(3) (2002), 213-259.

[3] E. Acerbi And G. Mingione, Gradient estimates for the $p(x)$ Laplacean system, J. Reine Angew. Math. 584 (2005), 117-148.

[4] E. Acerbi And G. Mingione, Gradient estimates for a class of parabolic systems, Duke Math. J. 136(2) (2007), 285-320.

[5] E. Acerbi, G. Mingione, And G. A. Seregin, Regularity results for parabolic systems related to a class of non-Newtonian fluids, Ann. Inst. H. Poincaré Anal. Non Linéaire 21(1) (2004), 25-60.

[6] S. Antontsev And V. Zhikov, Higher integrability for parabolic equations of $p(x, t)$-Laplacian type, Adv. Differential Equations 10(9) (2005), 1053-1080. 
[7] V. BöGELEIN, Higher integrability for weak solutions of higher order degenerate parabolic systems, Ann. Acad. Sci. Fenn. Math. 33(2) (2008), 387-412.

[8] V. BöGELEIN, Very weak solutions of higher-order degenerate parabolic systems, Adv. Differential Equations 14(1-2) (2009), 121-200.

[9] V. Bögelein, F. Duzaar, and G. Mingione, The boundary regularity of non-linear parabolic systems. II, Ann. Inst. H. Poincaré Anal. Non Linéaire 27(1) (2010), 145-200.

[10] V. Bögelein, F. Duzaar, and G. Mingione, The regularity of general parabolic systems with degenerate diffusions, Preprint (2010).

[11] V. BöGelein And M. Parviainen, Self-improving property of nonlinear higher order parabolic systems near the boundary, NoDEA Nonlinear Differential Equations Appl. 17(1) (2010), 21-54.

[12] V. BöGelein and A. Zatorska-Goldstein, Higher integrability of very weak solutions of systems of $p(x)$-Laplacean type, J. Math. Anal. Appl. 336(1) (2007), 480-497.

[13] A. Coscia And G. Mingione, Hölder continuity of the gradient of $p(x)$-harmonic mappings, C. R. Acad. Sci. Paris Sér. I Math. 328(4) (1999), 363-368.

[14] E. DiBenedetto, "Degenerate parabolic equations", Universitext, Springer-Verlag, New York, 1993.

[15] E. DiBenedetto And A. Friedman, Regularity of solutions of nonlinear degenerate parabolic systems, J. Reine Angew. Math. 349 (1984), 83-128.

[16] E. DiBenedetto And A. Friedman, Hölder estimates for nonlinear degenerate parabolic systems, J. Reine Angew. Math. 357 (1985), 1-22.

[17] E. DiBenedetto And A. Friedman, Addendum to: "Hölder estimates for nonlinear degenerate parabolic systems", J. Reine Angew. Math. 363 (1985), 217-220.

[18] E. DiBenedetto And J. Manfredi, On the higher integrability of the gradient of weak solutions of certain degenerate elliptic systems, Amer. J. Math. 115(5) (1993), 1107-1134.

[19] F. Duzana, G. Mingione, and K. Steffen, Parabolic systems with polynomial growth and regularity, Mem. Amer. Math. Soc. (to appear). 
[20] L. C. Evans And R. F. Gariepy, "Measure theory and fine properties of functions", Studies in Advanced Mathematics, CRC Press, Boca Raton, FL, 1992.

[21] F. W. GeHring, The $L^{p}$-integrability of the partial derivatives of a quasiconformal mapping, Acta Math. 130 (1973), 265-277.

[22] M. Giaquinta, "Multiple integrals in the calculus of variations and nonlinear elliptic systems", Annals of Mathematics Studies 105, Princeton University Press, Princeton, NJ, 1983.

[23] M. Giaquinta and G. Modica, Regularity results for some classes of higher order nonlinear elliptic systems, J. Reine Angew. Math. 311/312 (1979), 145-169.

[24] M. Giaquinta and M: Struwe, On the partial regularity of weak solutions of nonlinear parabolic systems, Math. Z. 179(4) (1982), 437-451.

[25] P. HaRjulehto ANd P. Hästö, Lebesgue points in variable exponent spaces, Ann. Acad. Sci. Fenn. Math. 29(2) (2004), 295-306.

[26] P. Harjulehto, P. Hëstö, and V. Latvala, Lebesgue points in variable exponent Sobolev spaces on metric measure spaces, in: "Complex Analysis and Free Boundary Flows", Transactions of the Institute of Mathematics of the National Academy of Sciences of Ukraine 1(3), 2004, pp. 87-99.

[27] T. Iwaniec, Projections onto gradient fields and $L^{p}$-estimates for degenerated elliptic operators, Studia Math. 75(3) (1983), 293-312.

[28] T. Iwaniec and C. Sbordone, Weak minima of variational integrals, J. Reine Angew. Math. 454 (1994), 143-161.

[29] J. Kinnunen and J. L. Lewis, Higher integrability for parabolic systems of $p$-Laplacian type, Duke Math. J. 102(2) (2000), 253-271.

[30] J. Kinnunen and J. L. Lewis, Very weak solutions of parabolic systems of $p$-Laplacian type, Ark. Mat. 40(1) (2002), 105-132.

[31] J. Kinnunen and S. Zhou, A local estimate for nonlinear equations with discontinuous coefficients, Comm. Partial Differential Equations 24(11-12) (1999), 2043-2068.

[32] J. Kinnunen And S. Zhou, A boundary estimate for nonlinear equations with discontinuous coefficients, Differential Integral Equations 14(4) (2001), 475-492.

[33] J. L. LEWIS, On very weak solutions of certain elliptic systems, Comm. Partial Differential Equations 18(9-10) (1993), 1515-1537.

[34] N. G. Meyers, An $L^{p}$-estimate for the gradient of solutions of second order elliptic divergence equations, Ann. Scuola Norm. Sup. Pisa (3) 17 (1963), 189-206. 
[35] N. G. Meyers and A. Elcrat, Some results on regularity for solutions of non-linear elliptic systems and quasi-regular functions, Duke Math. J. 42 (1975), 121-136.

[36] G. Mingione, The Calderón-Zygmund theory for elliptic problems with measure data, Ann. Sc. Norm. Super. Pisa Cl. Sci. (5) 6(2) (2007), 195-261.

[37] L. Nirenberg, On elliptic partial differential equations, Ann. Scuola Norm. Sup. Pisa (3) 13 (1959), 115-162.

[38] M. Parviainen, Global gradient estimates for degenerate parabolic equations in nonsmooth domains, Ann. Mat. Pura Appl. (4) 188(2) (2009), 333-358.

[39] C. Scheven, Regularity for subquadratic parabolic systems: higher integrability and dimension estimates, Proc. Roy. Soc. Edinburgh Sect. A 140(6) (2010), 1269-1308.

[40] K. UhlenBeCK, Regularity for a class of non-linear elliptic systems, Acta Math. 138(3-4) (1977), 219-240.

[41] V. V. Zhikov, On some variational problems, Russian J. Math. Phys. 5(1) (1997), 105-116 (1998).

$[42]$ V. V. ZhiKov and S. E. PAstukhova, On the improved integrability of the gradient of solutions of elliptic equations with a variable nonlinearity exponent, (Russian), Mat. Sb. 199(12) (2008), 19-52; translation in: Sb. Math. 199(11-12) (2008), 1751-1782.

Department Mathematik

Universität Erlangen-Nürnberg

Bismarckstrasse 1 1/2

91054 Erlangen

Germany

E-mail address: boegelein@mi.uni-erlangen.de

E-mail address: duzaar@mi.uni-erlangen.de

Primera versió rebuda el 9 d'abril de 2010, darrera versió rebuda el 27 de setembre de 2010 . 